Supplementary Material

\title{
Effects of 2,3-Dehydrosilybin, its Galloyl Ester and Methyl Ether Derivatives on Human Umbilical Vein Endothelial Cells
}

Daniel Karas, ${ }^{\dagger}, *$ Radek Gažák, ${ }^{\S}$ Kateřina Valentová, ${ }^{\dagger,},{ }^{*}$ Christopher S. Chambers, ${ }^{\S}$ Veronika Pivodová, ${ }^{\dagger, \ddagger}$ David Biedermann, ${ }^{\S}$ Alena Křenková, ${ }^{\S}$ Ivana Oborná, ${ }^{\perp}$ Marek Kuzma, ${ }^{\S}$ Josef Cvačka," Jitka Ulrichová, ${ }^{\dagger, *}$ Vladimír Křen ${ }^{\S}$

${ }^{\dagger}$ Department of Medical Chemistry and Biochemistry, Faculty of Medicine and Dentistry, Palacký University, Hněvotínská 3, CZ-775 15 Olomouc, Czech Republic

$\$$ Institute of Molecular and Translational Medicine, Faculty of Medicine and Dentistry, Palacký University, Hněvotínská 3, CZ-775 15 Olomouc, Czech Republic

${ }^{\S}$ Institute of Microbiology, Czech Academy of Sciences, Vídeňská 1083, Prague 4, CZ-142 20, Czech Republic

${ }^{\perp}$ Department of Obstetrics and Gynecology, Faculty of Medicine and Dentistry, Palacký University and University Hospital, I.P. Pavlova 6, CZ-775 20 Olomouc, Czech Republic

" Institute of Organic Chemistry and Biochemistry, Czech Academy of Sciences, Flemingovo nám. 2, CZ-16610 Prague 6, Czech Republic

*Corresponding author. Tel.: +420 296442 509. E-mail: kata.valentova@email.cz (K. Valentová). 


\section{Table of contents}

Figure S1. ${ }^{1} \mathrm{H}$ NMR spectrum (600.23 MHz, 303.2 K, DMSO- $d_{6}$ ) of 7-O-benzyl-3-O-[(3',4',5'-tri-O-benzyl)galloyl]-2,3dehydrosilybin (10)........

Figure S2: ${ }^{13} \mathrm{C}$ NMR spectrum (150.93 MHz, 303.2 K, DMSO- $\left.d_{6}\right)$ of 7-O-benzyl-3-O-[(3',4',5'-tri-O-benzyl)galloyl]-2,3dehydrosilybin (10)

Figure S3. ESI-MS- spectrum of 7-O-benzyl-3-O-[(3',4',5'-tri-O-benzyl)galloyl]-2,3-dehydrosilybin (10) .............................. 4

Figure S4. ${ }^{1} \mathrm{H}$ NMR spectrum $\left(700.13 \mathrm{MHz}, 303.2 \mathrm{~K}\right.$, DMSO- $d_{6}$ ) of 3-O-galloyl-2,3-dehydrosilybin (11) ................................ 4

Figure S5. ${ }^{13} \mathrm{C}$ NMR spectrum $\left(176.05 \mathrm{MHz}, 303.2 \mathrm{~K}\right.$, DMSO-d $d_{6}$ ) of 3-O-galloyl-2,3-dehydrosilybin (11) .............................. 5

Figure S6. ESI-MS - spectrum of 3-O-galloyl-2,3-dehydrosilybin (11) ....................................................................... 5

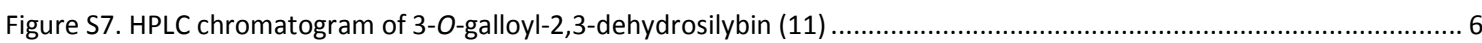

Figure S8. ${ }^{1} \mathrm{H}$ NMR spectrum $\left(600.23 \mathrm{MHz}, 303.2 \mathrm{~K}\right.$, DMSO- $\left.d_{6}\right)$ of 7-O-(3',4',5'-tri-O-benzylgalloyl)-2,3-dehydrosilybin (12) .. 6

Figure S9. ${ }^{13} \mathrm{C}$ NMR spectrum (150.93 MHz, 303.2 K, DMSO- $\left.d_{6}\right)$ of 7-O-(3', 4', 5'-tri-O-benzylgalloyl)-2,3-dehydrosilybin (12) 7

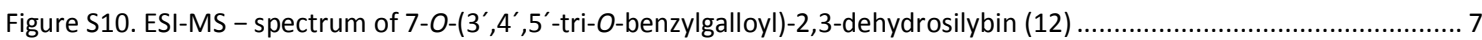

Figure S11. ${ }^{1} \mathrm{H}$ NMR spectrum $\left(600.23 \mathrm{MHz}, 303.2 \mathrm{~K}\right.$, DMSO- $d_{6}$ ) of 7-O-galloyl-2,3-dehydrosilybin (13) ............................... 8

Figure $\mathrm{S} 12 .{ }^{13} \mathrm{C}$ NMR spectrum $\left(150.93 \mathrm{MHz}, 303.2 \mathrm{~K}\right.$, DMSO-d $d_{6}$ ) of 7-O-galloyl-2,3-dehydrosilybin (13) ............................... 8

Figure S13. ESI-MS - spectrum of 7-O-galloyl-2,3-dehydrosilybin (13) ........................................................................ 9

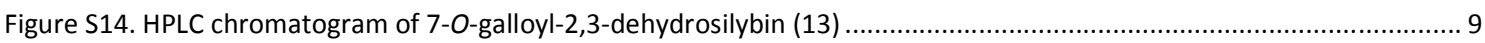

Figure S15. ${ }^{1} \mathrm{H}$ NMR spectrum (399.89 MHz, $303.2 \mathrm{~K}$, DMSO-d $d_{6}$ ) of 3,7-di-O-benzyl-2,3-dehydrosilybin (14) ....................... 10

Figure S16. ${ }^{13} \mathrm{C}$ NMR spectrum $\left(100.56 \mathrm{MHz}, 303.2 \mathrm{~K}\right.$, DMSO-d $\left.d_{6}\right)$ of 3,7-di-O-benzyl-2,3-dehydrosilybin (14) ....................... 10

Figure S17. ${ }^{1} \mathrm{H}$ NMR spectrum (600.23 MHz, 303.2 K, DMSO- $d_{6}$ ) of 3,7-di-O-benzyl-23-O-(tert-butyldimethylsilyl)-2,3-

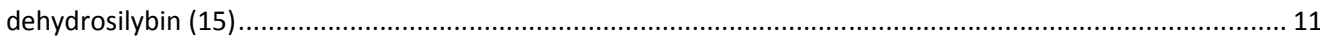

Figure S18. ${ }^{13} \mathrm{C}$ NMR spectrum (150.93 MHz, 303.2 K, DMSO- $d_{6}$ ) of 3,7-di-O-benzyl-23-O-(tert-butyldimethylsilyl)-2,3-

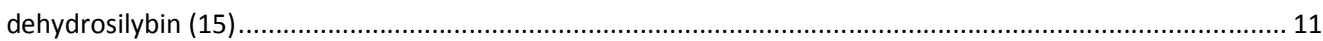

Figure S19. ESI-MS - spectrum of 3,7-di-O-benzyl-23-O-(tert-butyldimethylsilyl)-2,3-dehydrosilybin (15) ........................... 12

Figure S20. ${ }^{1} \mathrm{H}$ NMR spectrum $\left(700.13 \mathrm{MHz}, 303.2 \mathrm{~K}\right.$, DMSO- $\left.d_{6}\right)$ of 23-O-tert-butyldimethylsilyl-20-O-[(3', 4', $5^{\prime}$-tri-O-

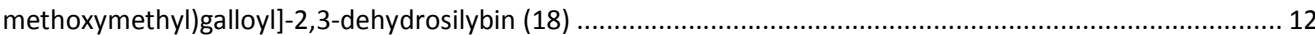

Figure S21. ${ }^{13} \mathrm{C}$ NMR spectrum $\left(176.05 \mathrm{MHz}, 303.2 \mathrm{~K}\right.$, DMSO- $\left.d_{6}\right)$ of 23-O-tert-butyldimethylsilyl-20-O-[(3',4',5'-tri-Omethoxymethyl)galloyl]-2,3-dehydrosilybin (18) .............................................................................. 13

Figure S22. ${ }^{1} \mathrm{H}$ NMR spectrum $\left(600.23 \mathrm{MHz}, 303.2 \mathrm{~K}\right.$, DMSO-d $\left.d_{6}\right)$ of 20-0-galloyl-2,3-dehydrosilybin (19) ........................... 13

Figure S23. ${ }^{13} \mathrm{C}$ NMR spectrum (150.93 MHz, $303.2 \mathrm{~K}$, DMSO-d $d_{6}$ ) of 20-O-galloyl-2,3-dehydrosilybin (19) ........................... 14

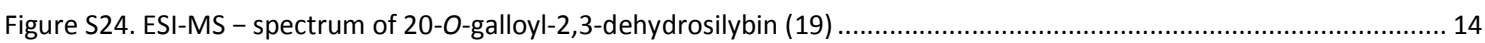

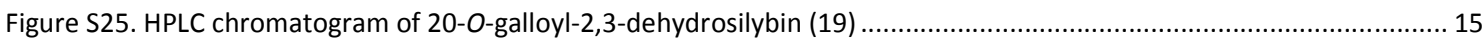

Figure S26. ${ }^{1} \mathrm{H}$ NMR spectrum (399.87 MHz, 303.2 K, DMSO- $d_{6}$ ) of 23-O-(3', 4', 5'-tri-O-methoxymethylgalloyl)-2,3-

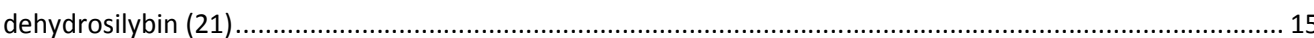

Figure S27. ${ }^{13} \mathrm{C}$ NMR spectrum $\left(100.56 \mathrm{MHz}, 303.2 \mathrm{~K}\right.$, DMSO- $\left.d_{6}\right)$ of 23-O-(3',4',5'-tri-O-methoxymethylgalloyl)-2,3-

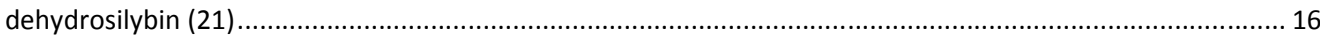

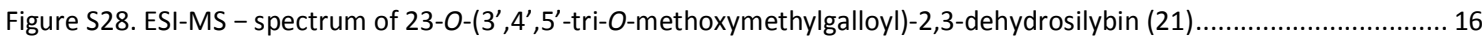

Figure S29. ${ }^{1} \mathrm{H}$ NMR spectrum (399.87 MHz, 303.2 K, DMSO- $d_{6}$ ) of 23-O-galloyl-2,3-dehydrosilybin (22) ........................... 17

Figure S30. ${ }^{13} \mathrm{C}$ NMR spectrum $\left(100.56 \mathrm{MHz}, 303.2 \mathrm{~K}\right.$, DMSO- $\left.d_{6}\right)$ of 23-O-galloyl-2,3-dehydrosilybin (22) ............................ 17

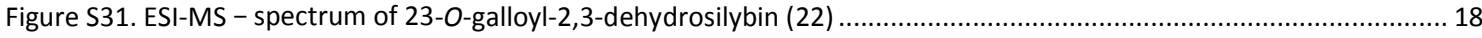

Figure S32. HPLC chromatogram of 23-O-galloyl-2,3-dehydrosilybin (22) ..................................................................... 18 


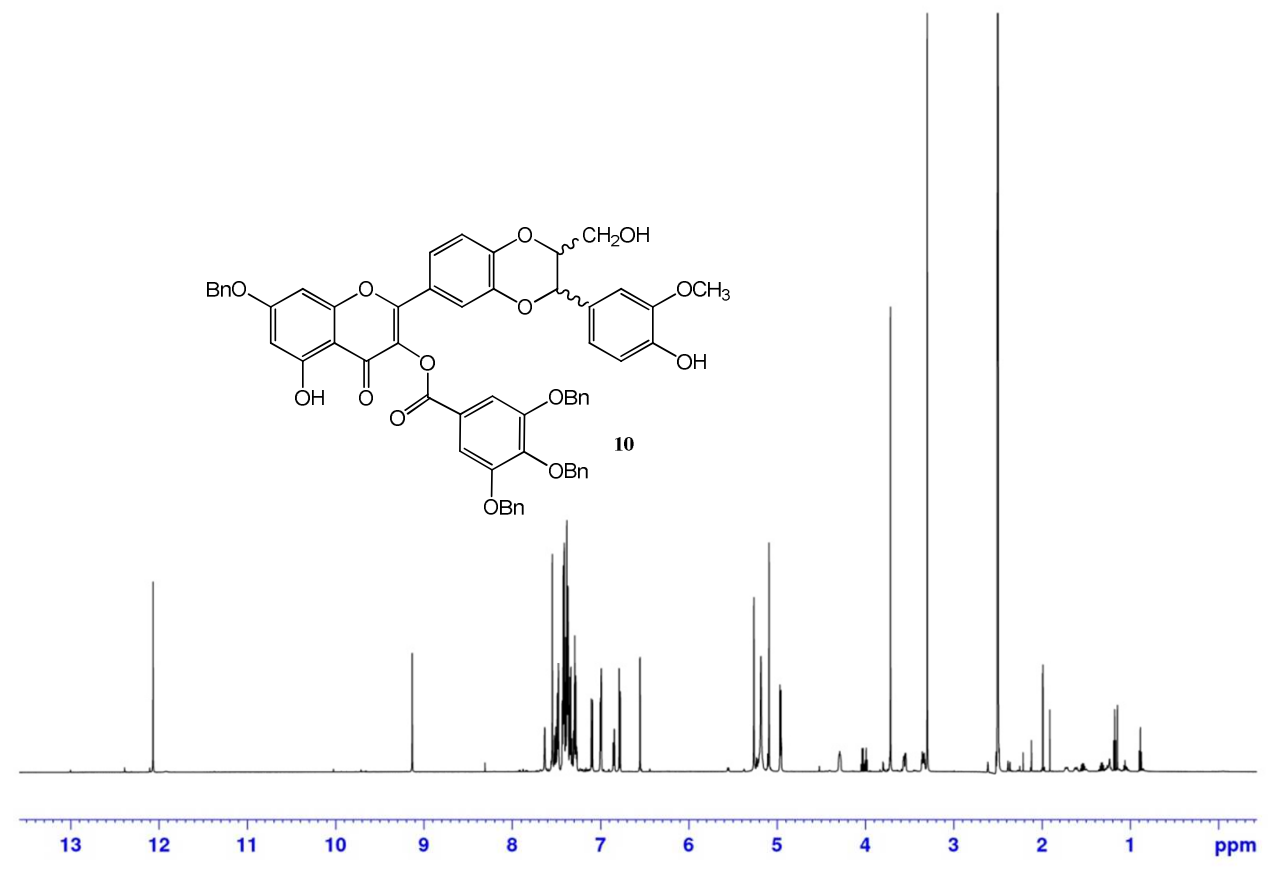

Figure S1. ${ }^{1} \mathrm{H}$ NMR spectrum $\left(600.23 \mathrm{MHz}, 303.2 \mathrm{~K}, \mathrm{DMSO}-\mathrm{d}_{6}\right)$ of 7-O-benzyl-3-O-[(3',4',5'-tri- $O$ benzyl)galloyl]-2,3-dehydrosilybin (10)

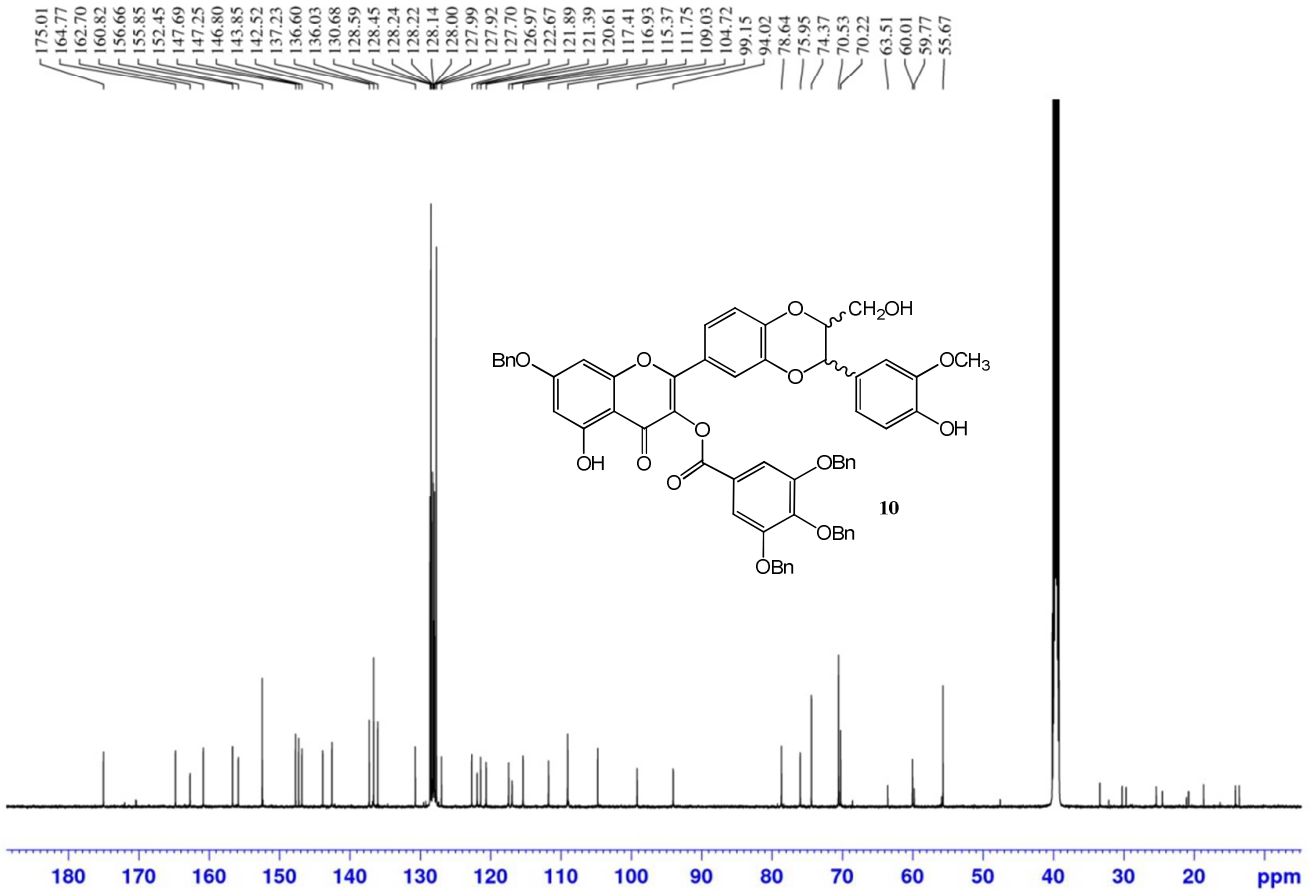

Figure S2: ${ }^{13} \mathrm{C}$ NMR spectrum $\left(150.93 \mathrm{MHz}, 303.2 \mathrm{~K}, \mathrm{DMSO}-\mathrm{d}_{6}\right)$ of 7- $O$-benzyl-3- $O$-[(3',4',5'-tri- $O$ benzyl)galloyl]-2,3-dehydrosilybin (10) 


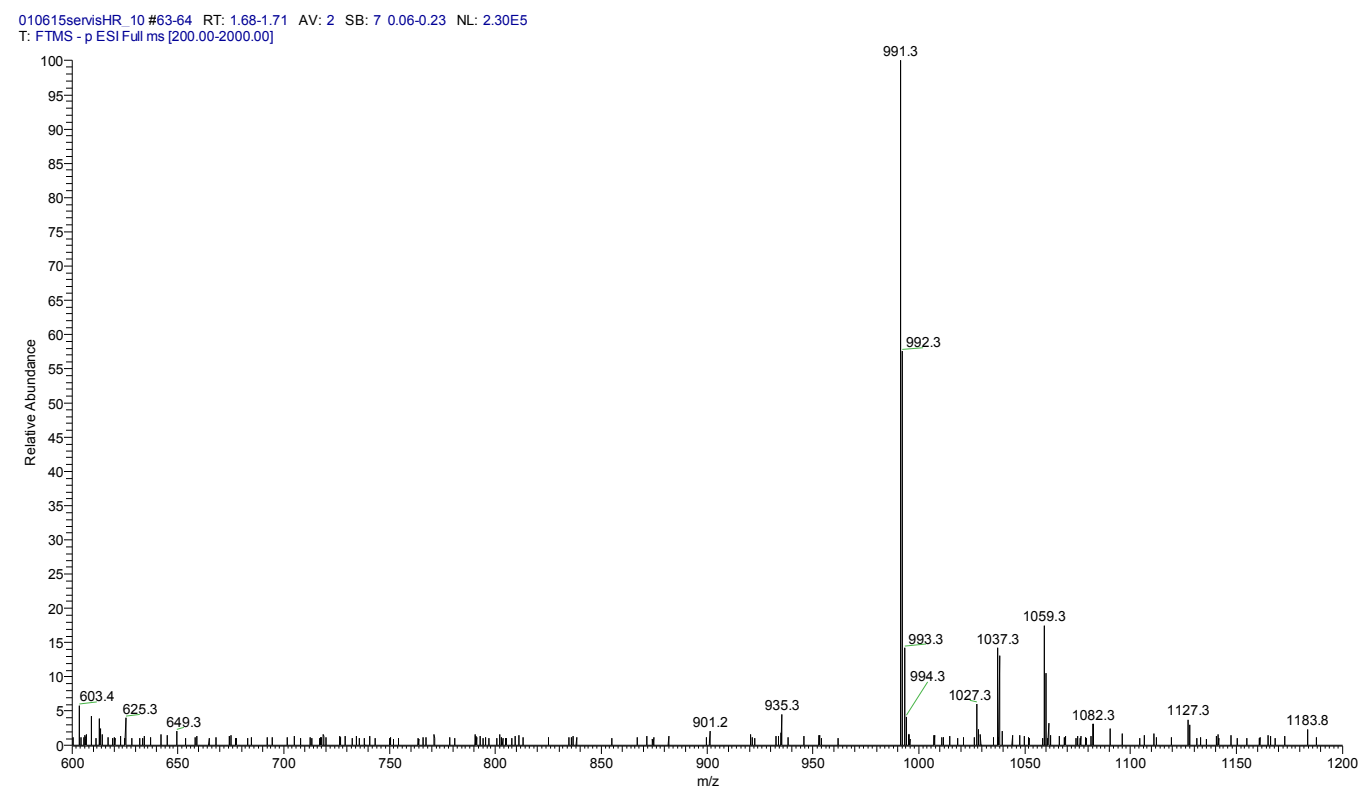

Figure S3. ESI-MS- spectrum of 7-O-benzyl-3-O-[(3',4',5'-tri-O-benzyl)galloyl]-2,3-dehydrosilybin (10)

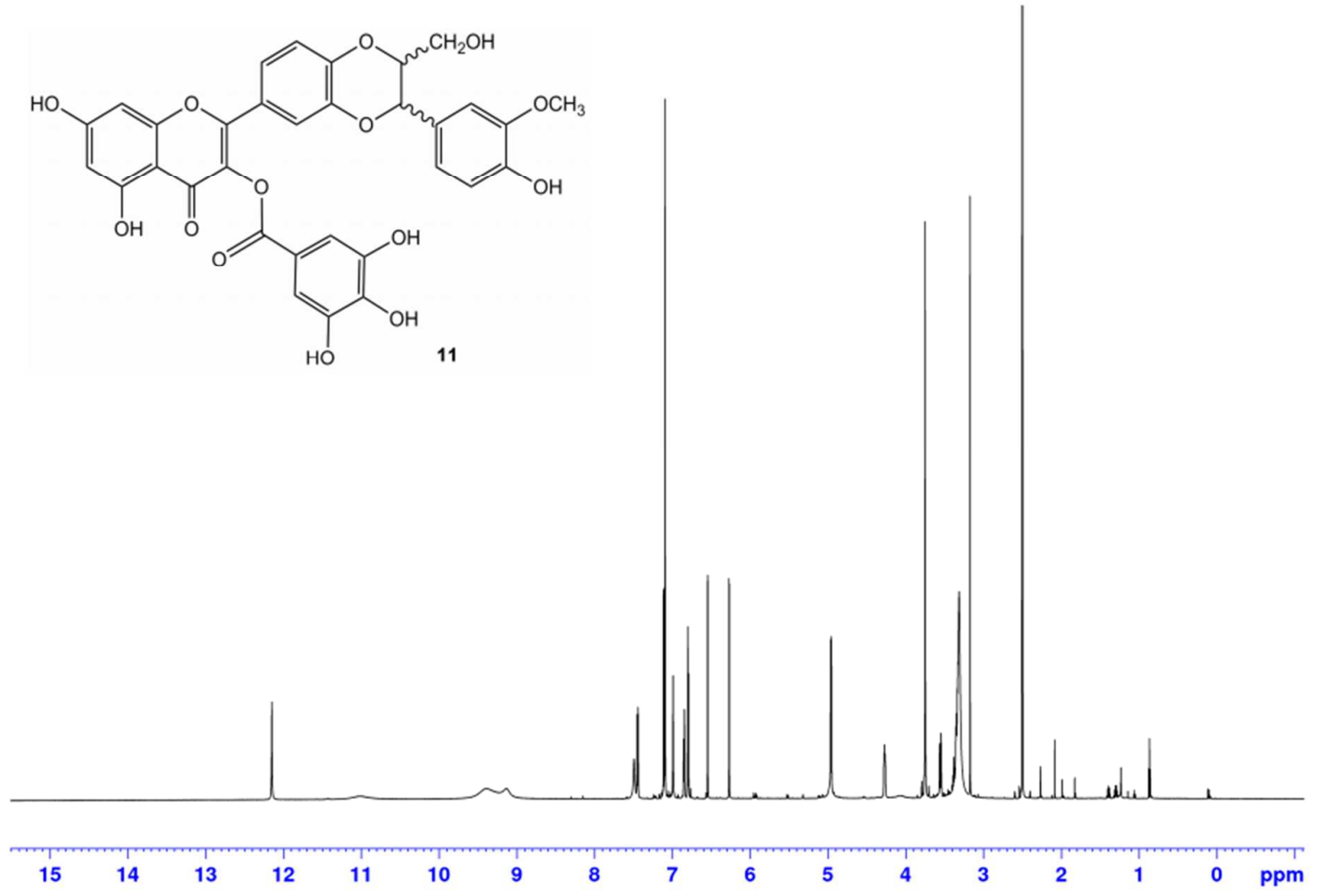

Figure S4. ${ }^{1} \mathrm{H}$ NMR spectrum $\left(700.13 \mathrm{MHz}, 303.2 \mathrm{~K}\right.$, DMSO- $\left.d_{6}\right)$ of 3-O-galloyl-2,3-dehydrosilybin (11) 


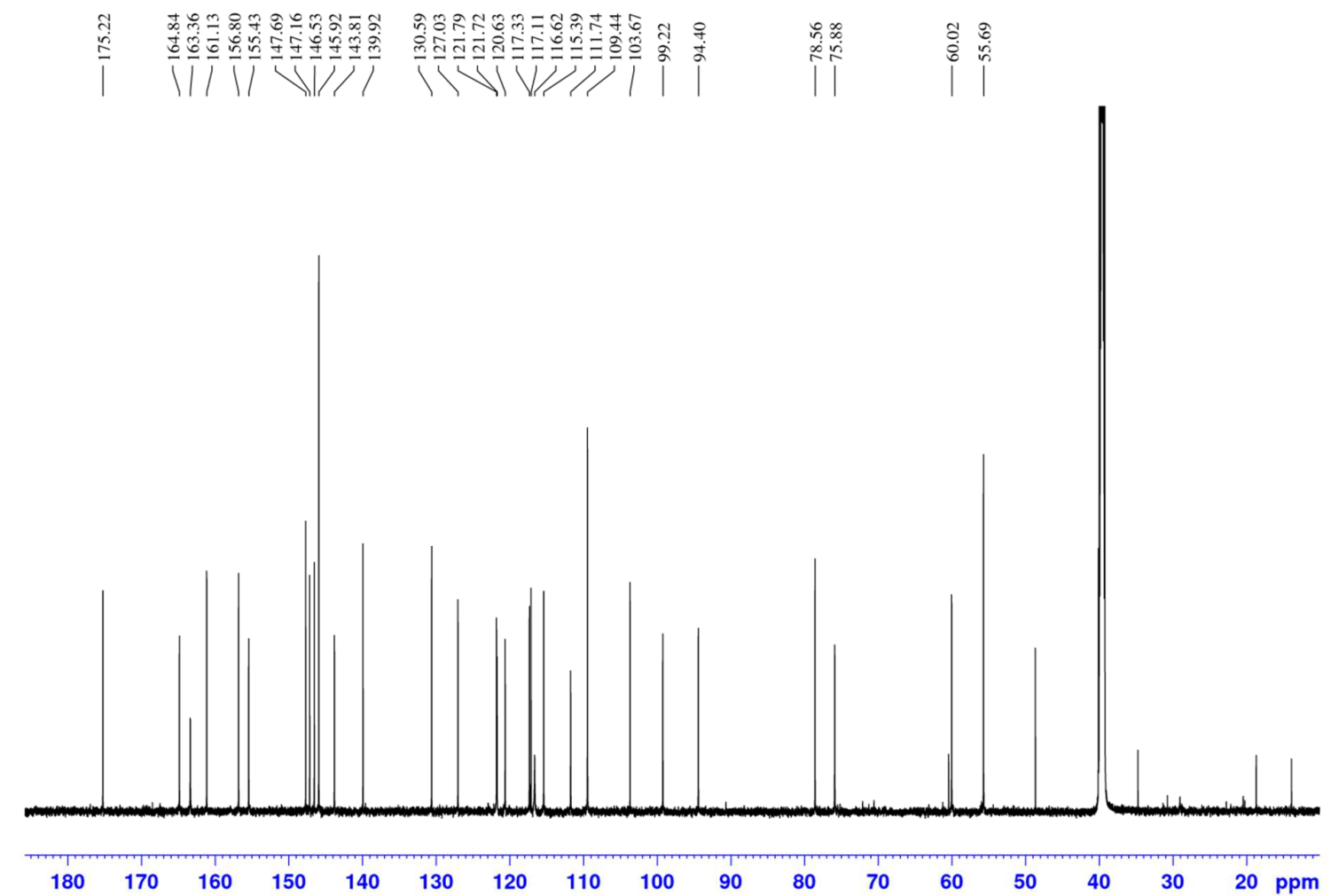

Figure S5. ${ }^{13} \mathrm{C}$ NMR spectrum (176.05 MHz, 303.2 K, DMSO- $\left.d_{6}\right)$ of 3-O-galloyl-2,3-dehydrosilybin (11)

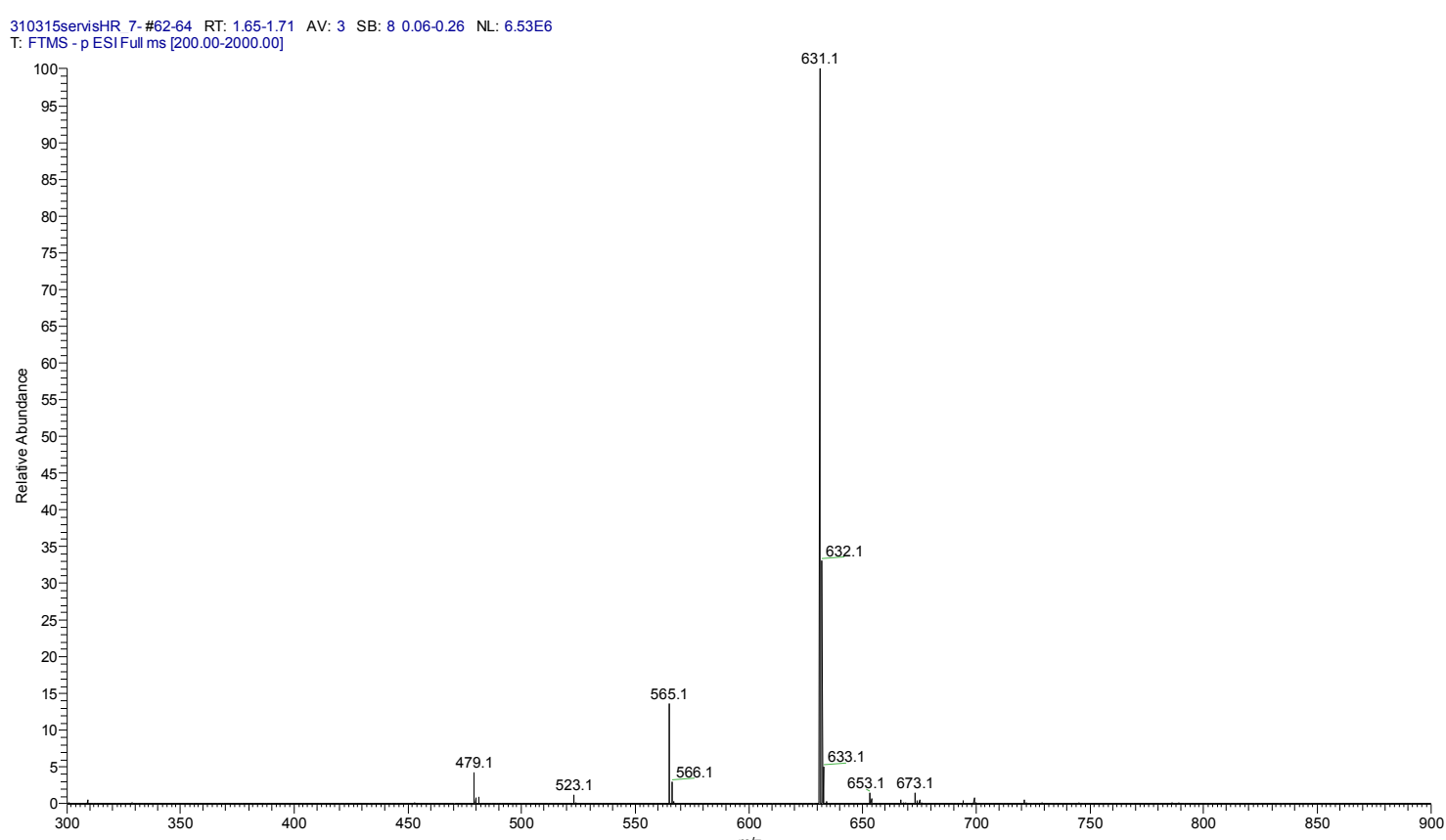

Figure S6. ESI-MS - spectrum of 3-O-galloyl-2,3-dehydrosilybin (11) 


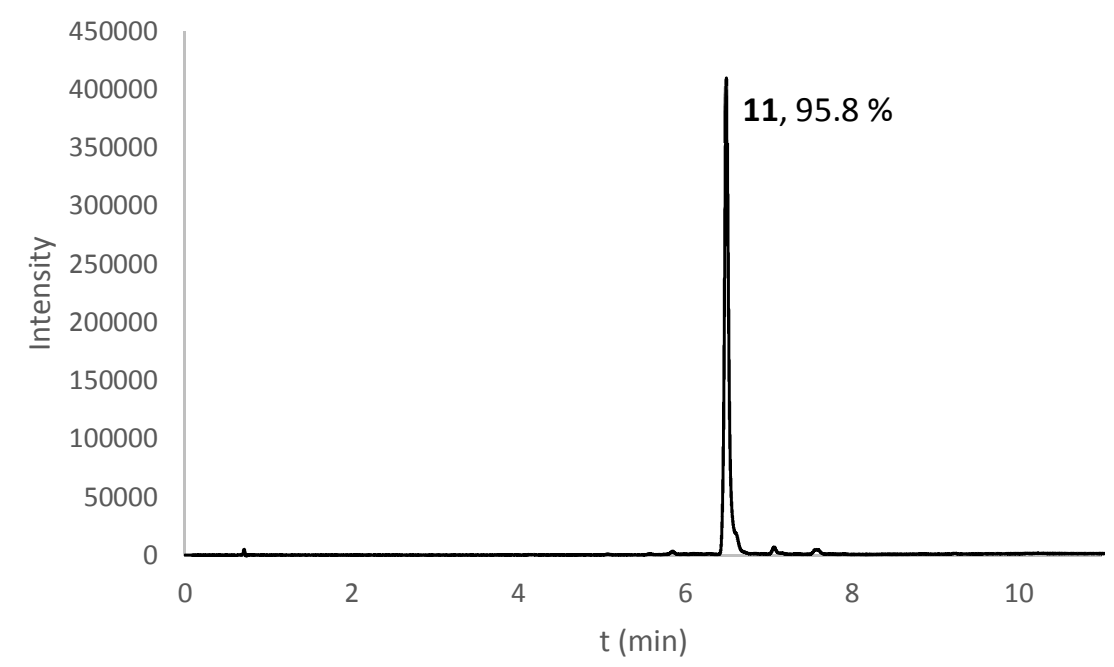

Figure S7. HPLC chromatogram of 3-0-galloyl-2,3-dehydrosilybin (11)

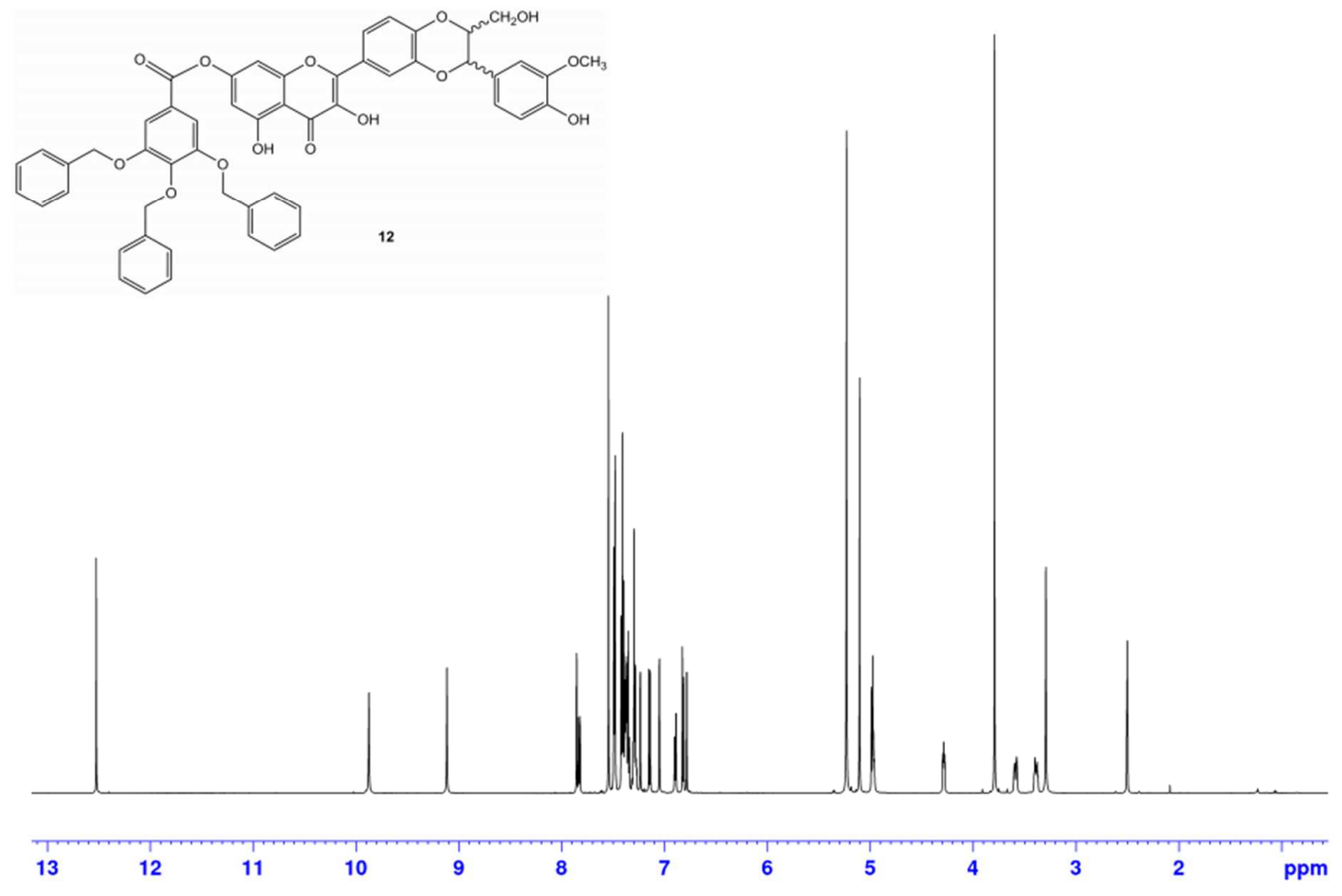

Figure S8. ${ }^{1} \mathrm{H}$ NMR spectrum $\left(600.23 \mathrm{MHz}, 303.2 \mathrm{~K}\right.$, DMSO- $\left.d_{6}\right)$ of 7-O-(3',4',5'-tri-O-benzylgalloyl)-2,3dehydrosilybin (12) 


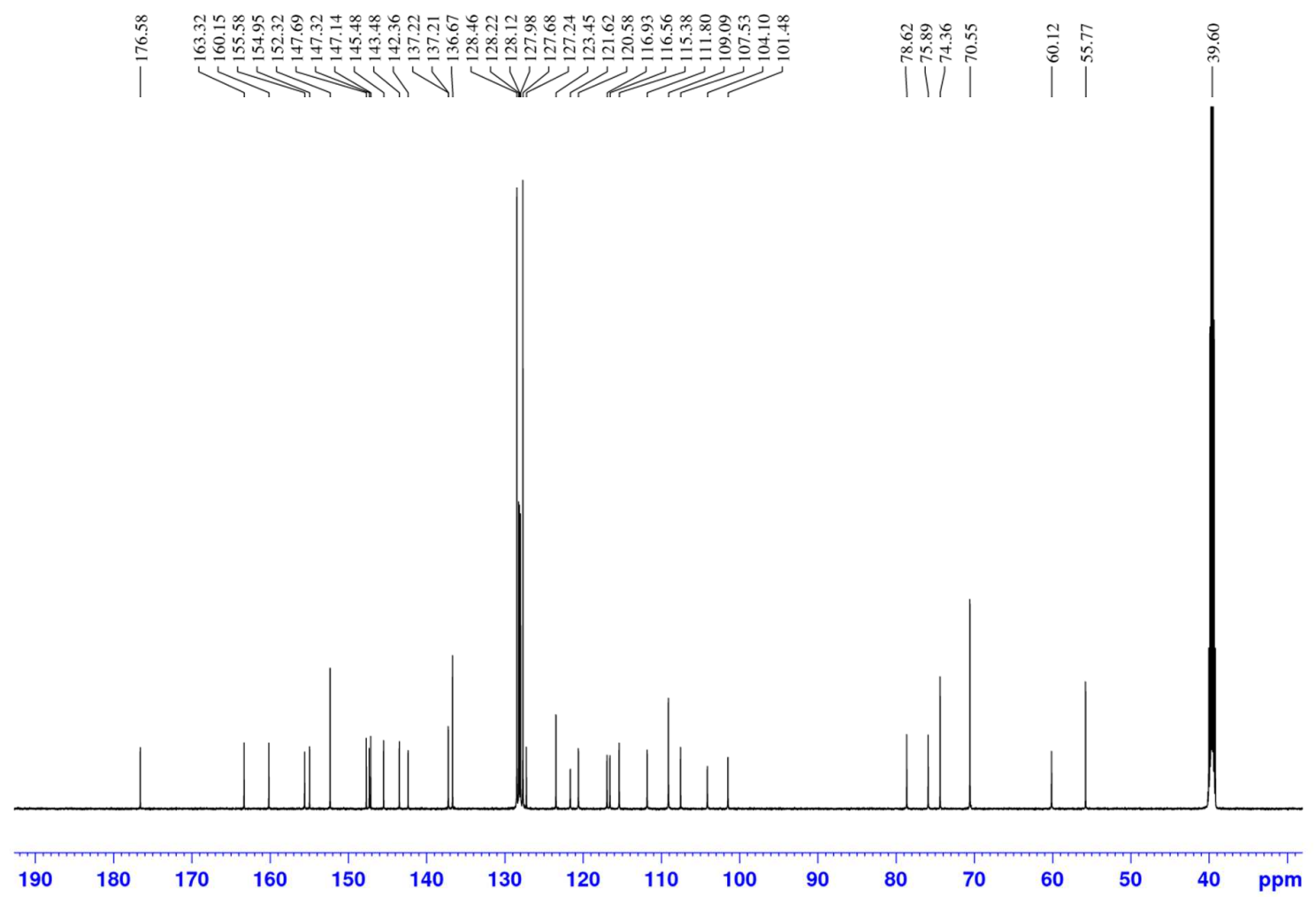

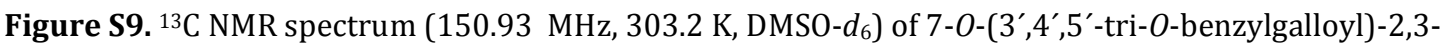
dehydrosilybin (12)

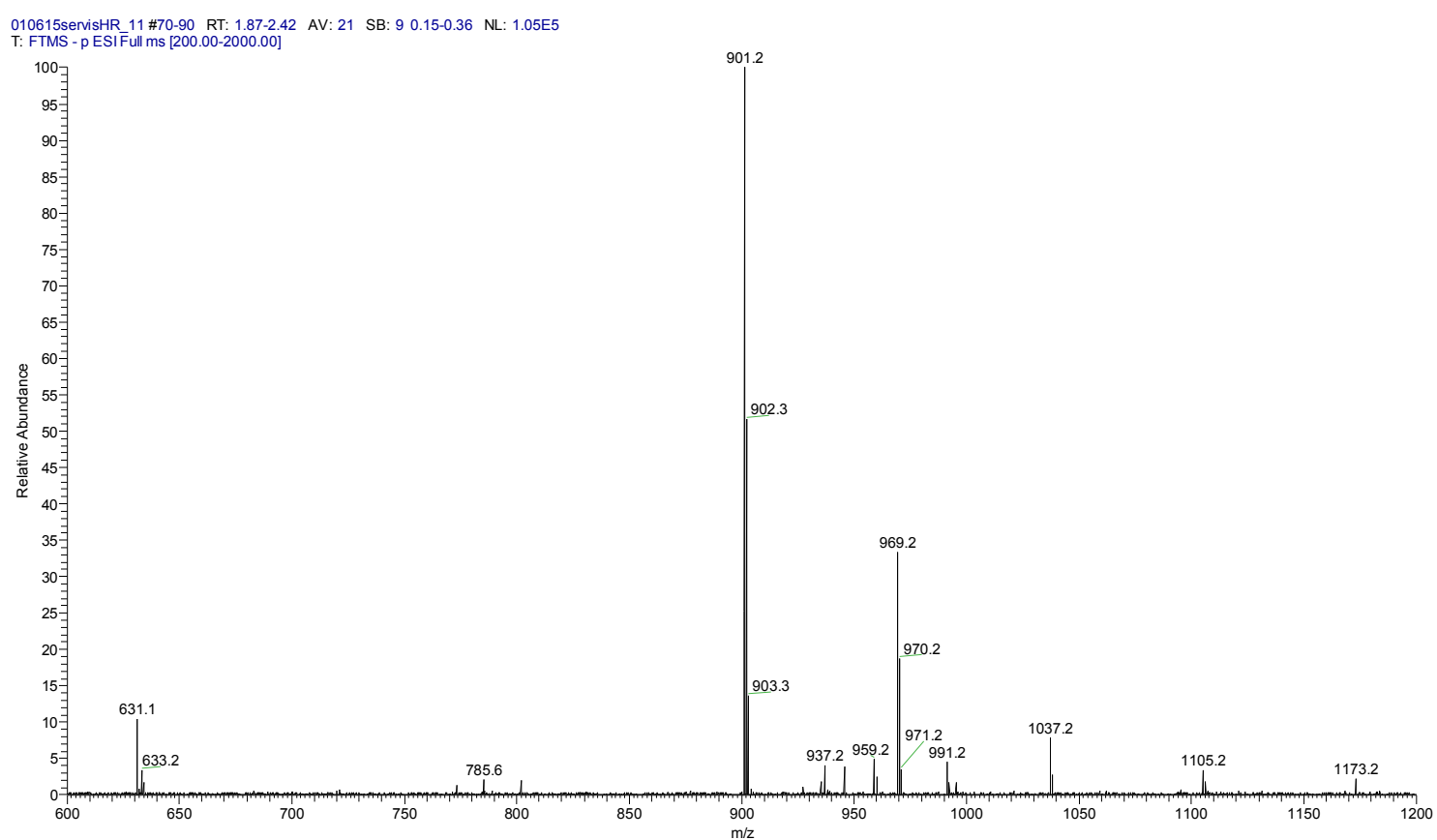

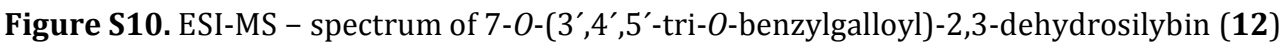




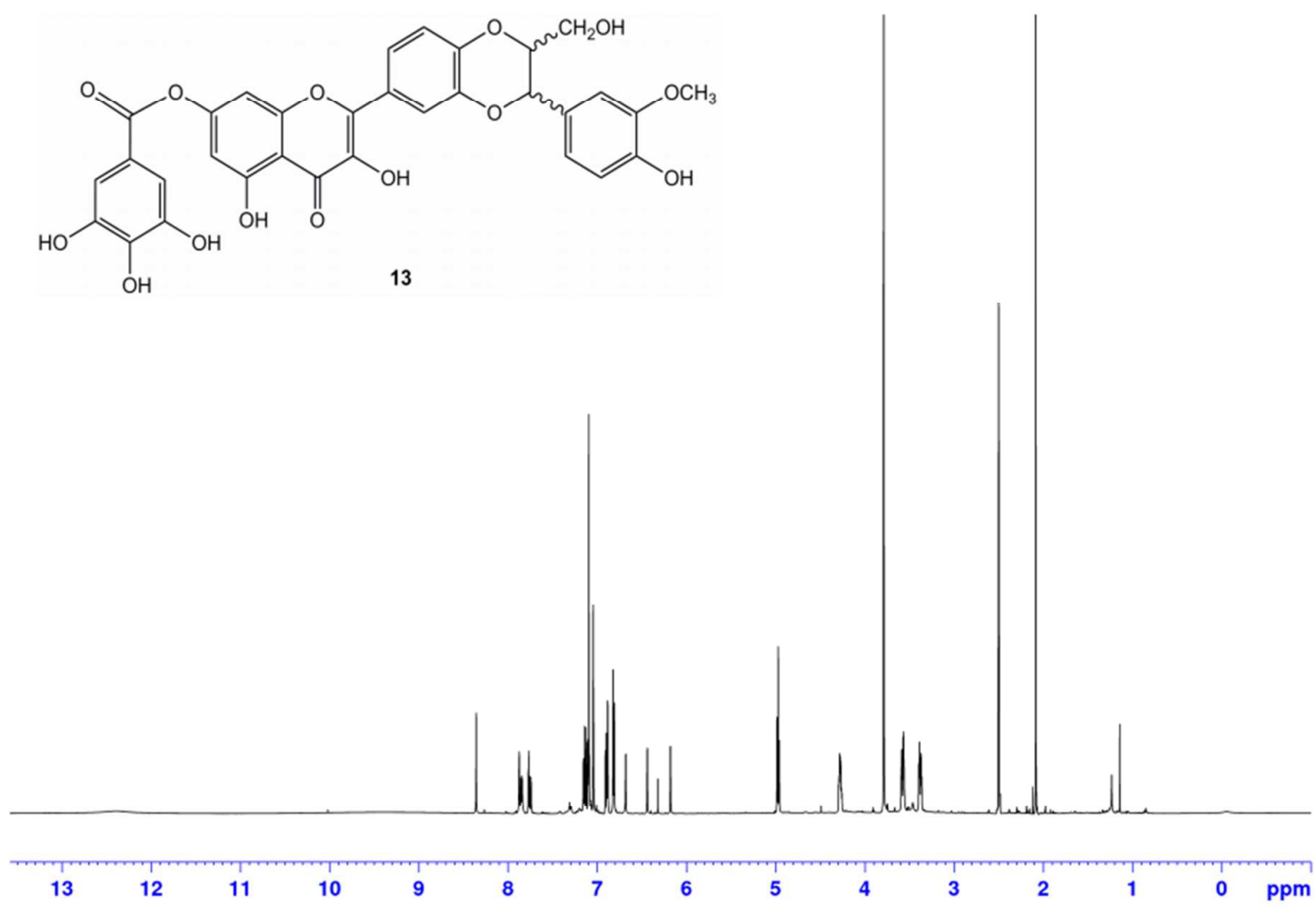

Figure S11. ${ }^{1} \mathrm{H}$ NMR spectrum $\left(600.23 \mathrm{MHz}, 303.2 \mathrm{~K}\right.$, DMSO- $\left.d_{6}\right)$ of 7-O-galloyl-2,3-dehydrosilybin (13)
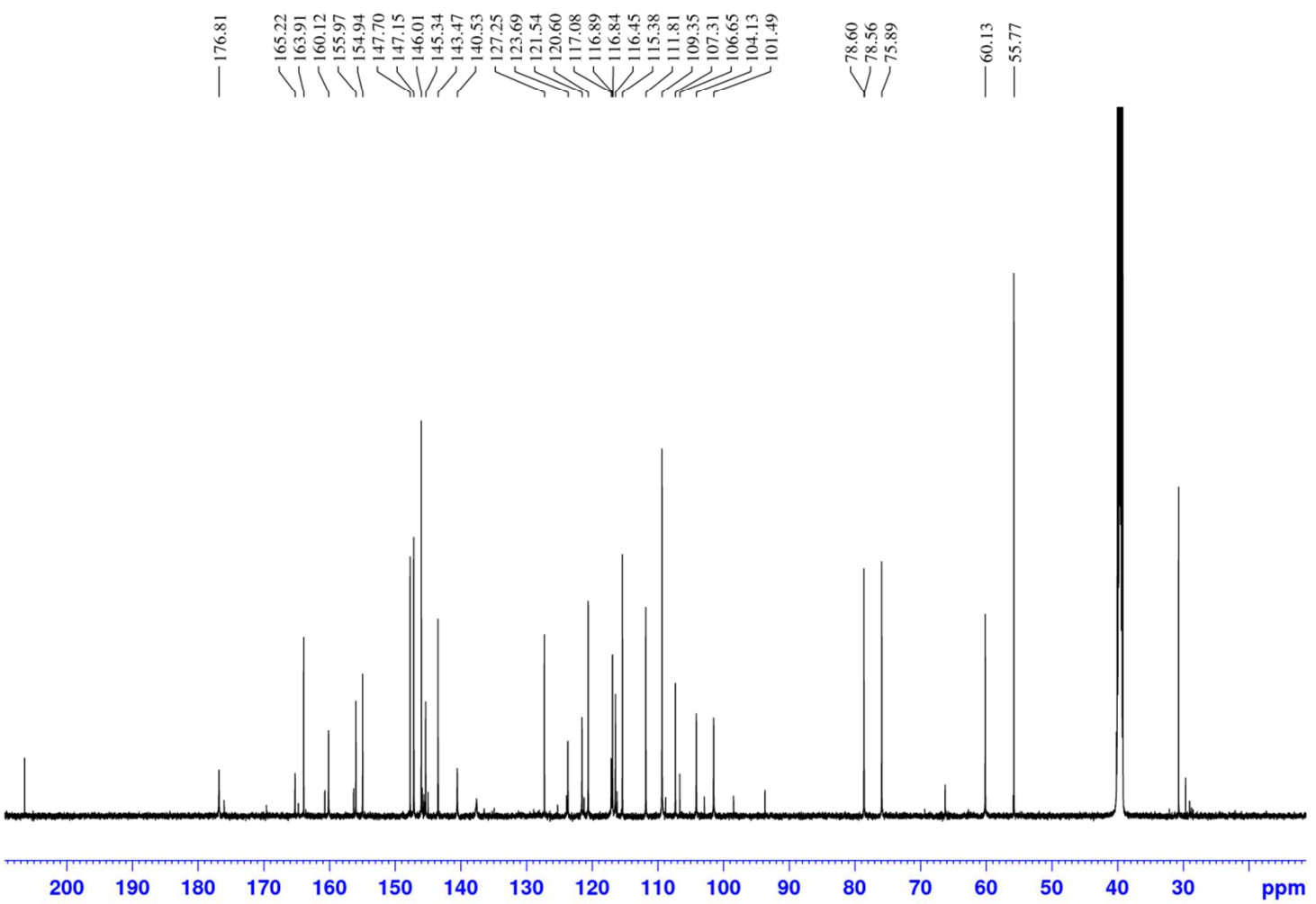

Figure S12. ${ }^{13} \mathrm{C}$ NMR spectrum $\left(150.93 \mathrm{MHz}, 303.2 \mathrm{~K}, \mathrm{DMSO}-d_{6}\right)$ of 7-O-galloyl-2,3-dehydrosilybin (13) 


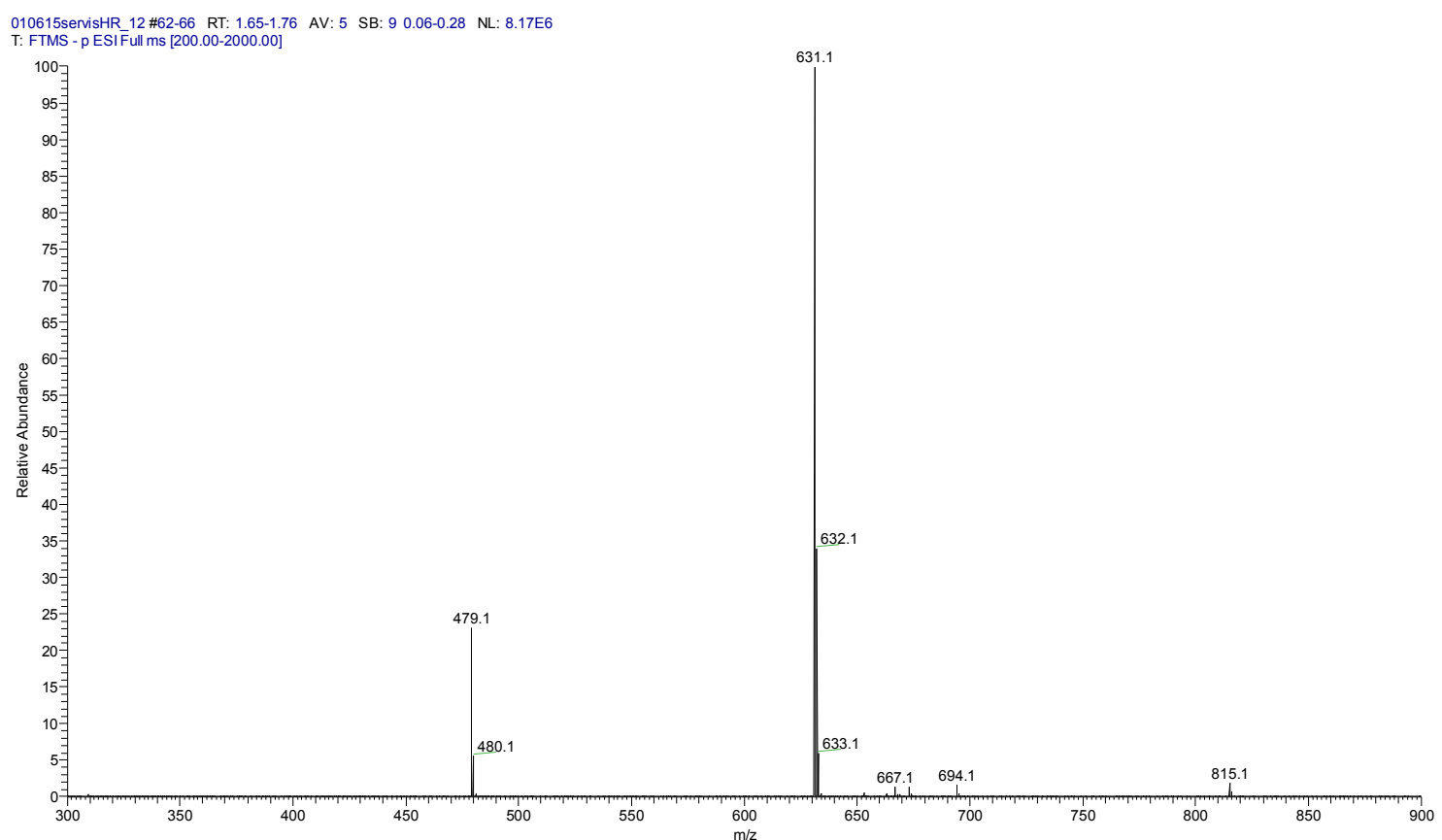

Figure S13. ESI-MS - spectrum of 7-O-galloyl-2,3-dehydrosilybin (13)

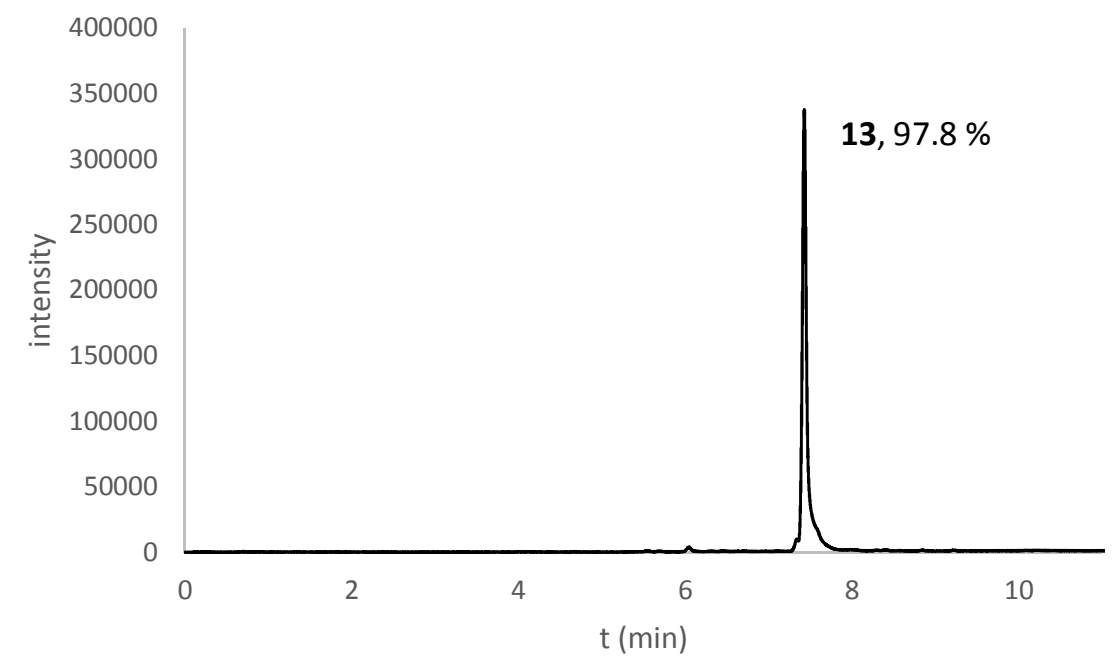

Figure S14. HPLC chromatogram of 7-O-galloyl-2,3-dehydrosilybin (13) 

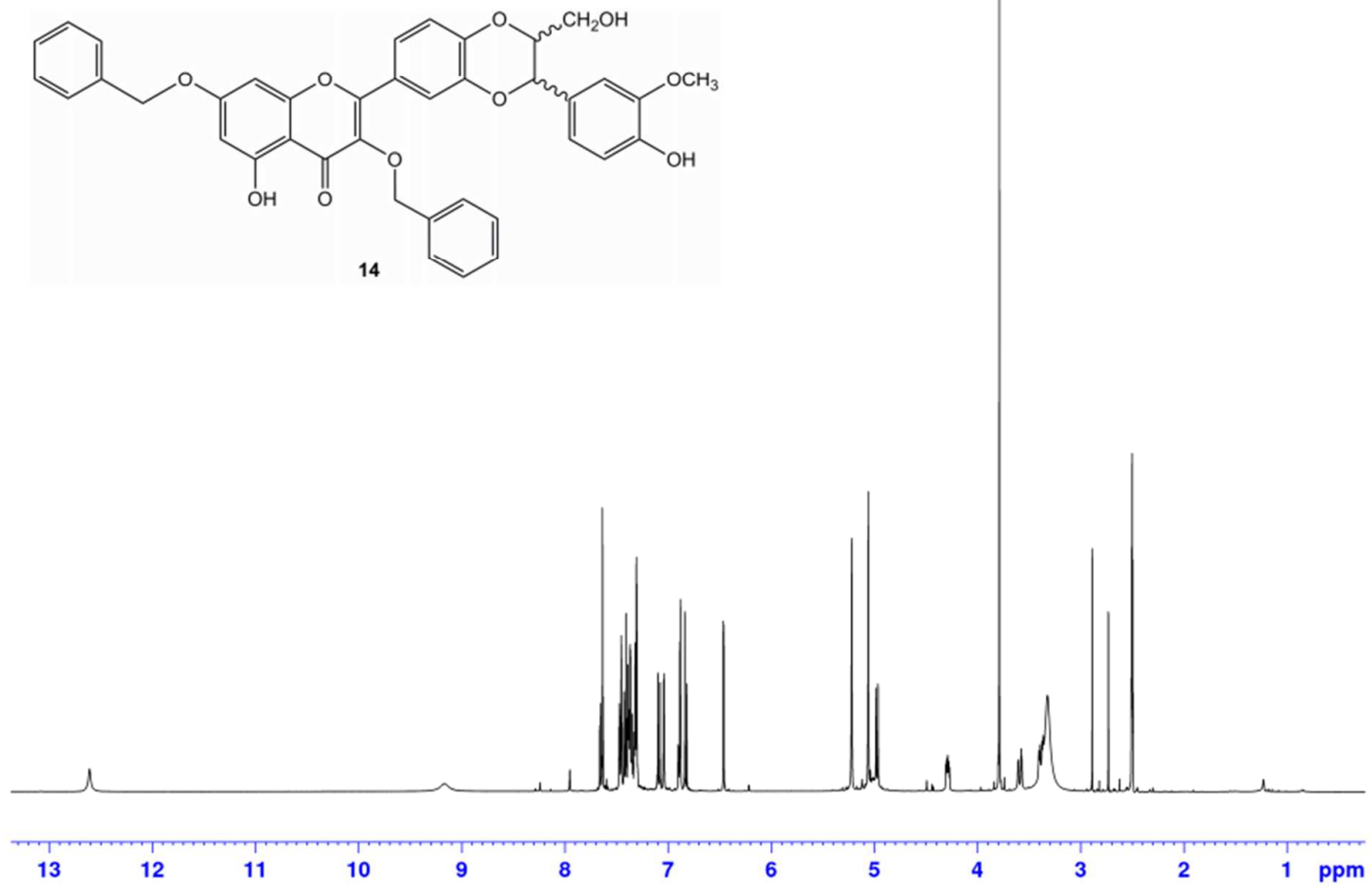

Figure S15. ${ }^{1} \mathrm{H}$ NMR spectrum $\left(399.89 \mathrm{MHz}, 303.2 \mathrm{~K}, \mathrm{DMSO}-\mathrm{d}_{6}\right.$ ) of 3,7-di- $O$-benzyl-2,3-dehydrosilybin (14)

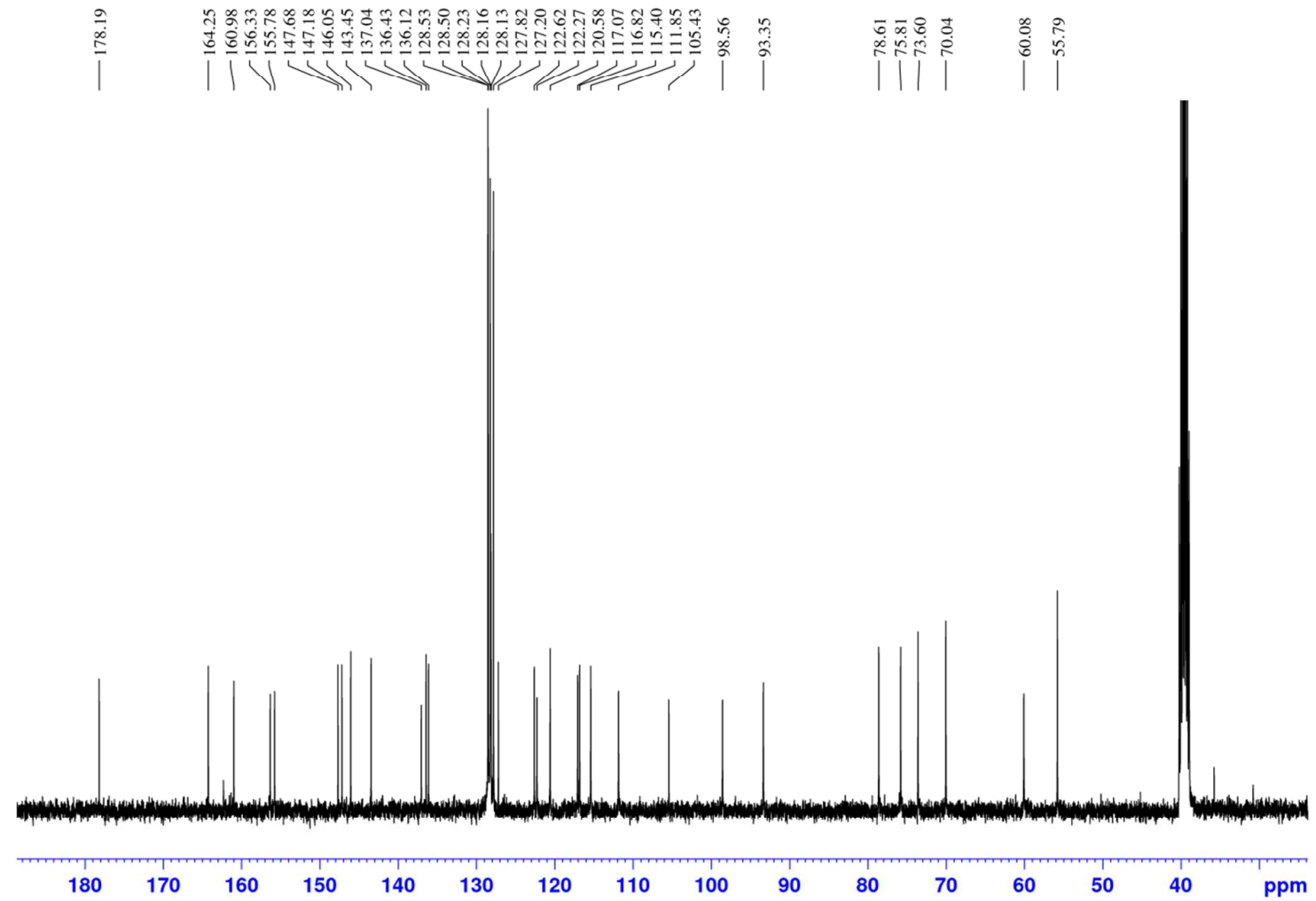

Figure S16. ${ }^{13} \mathrm{C}$ NMR spectrum $\left(100.56 \mathrm{MHz}, 303.2 \mathrm{~K}\right.$, DMSO- $d_{6}$ ) of 3,7-di- $O$-benzyl-2,3-dehydrosilybin (14) 


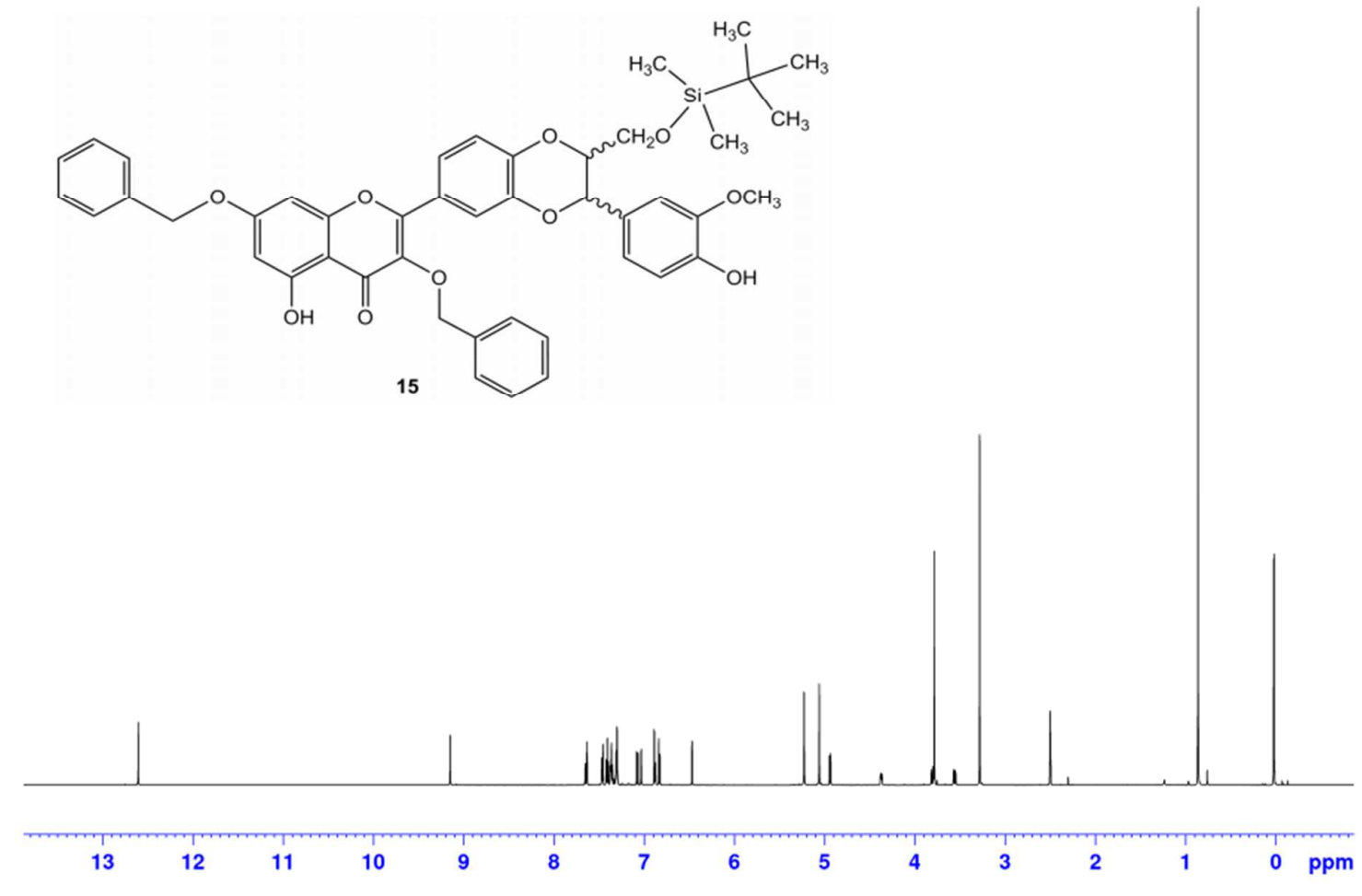

Figure S17. ${ }^{1} \mathrm{H}$ NMR spectrum $\left(600.23 \mathrm{MHz}, 303.2 \mathrm{~K}, \mathrm{DMSO}-d_{6}\right)$ of 3,7-di- $O$-benzyl-23-O-(tertbutyldimethylsilyl)-2,3-dehydrosilybin (15)

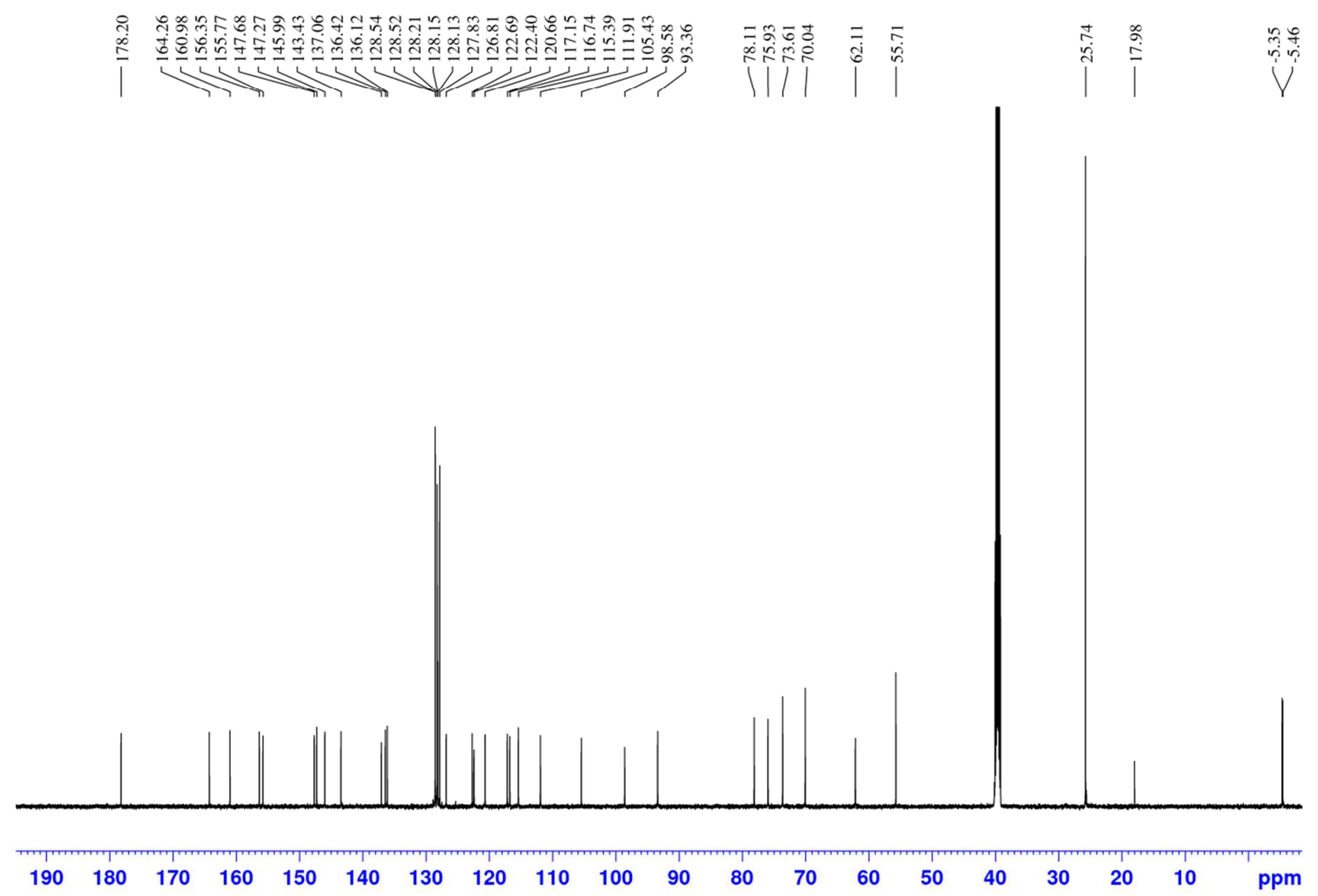

Figure S18. ${ }^{13} \mathrm{C}$ NMR spectrum $\left(150.93 \mathrm{MHz}, 303.2 \mathrm{~K}\right.$, DMSO- $\left.d_{6}\right)$ of 3,7-di-O-benzyl-23-O-(tertbutyldimethylsilyl)-2,3-dehydrosilybin (15) 


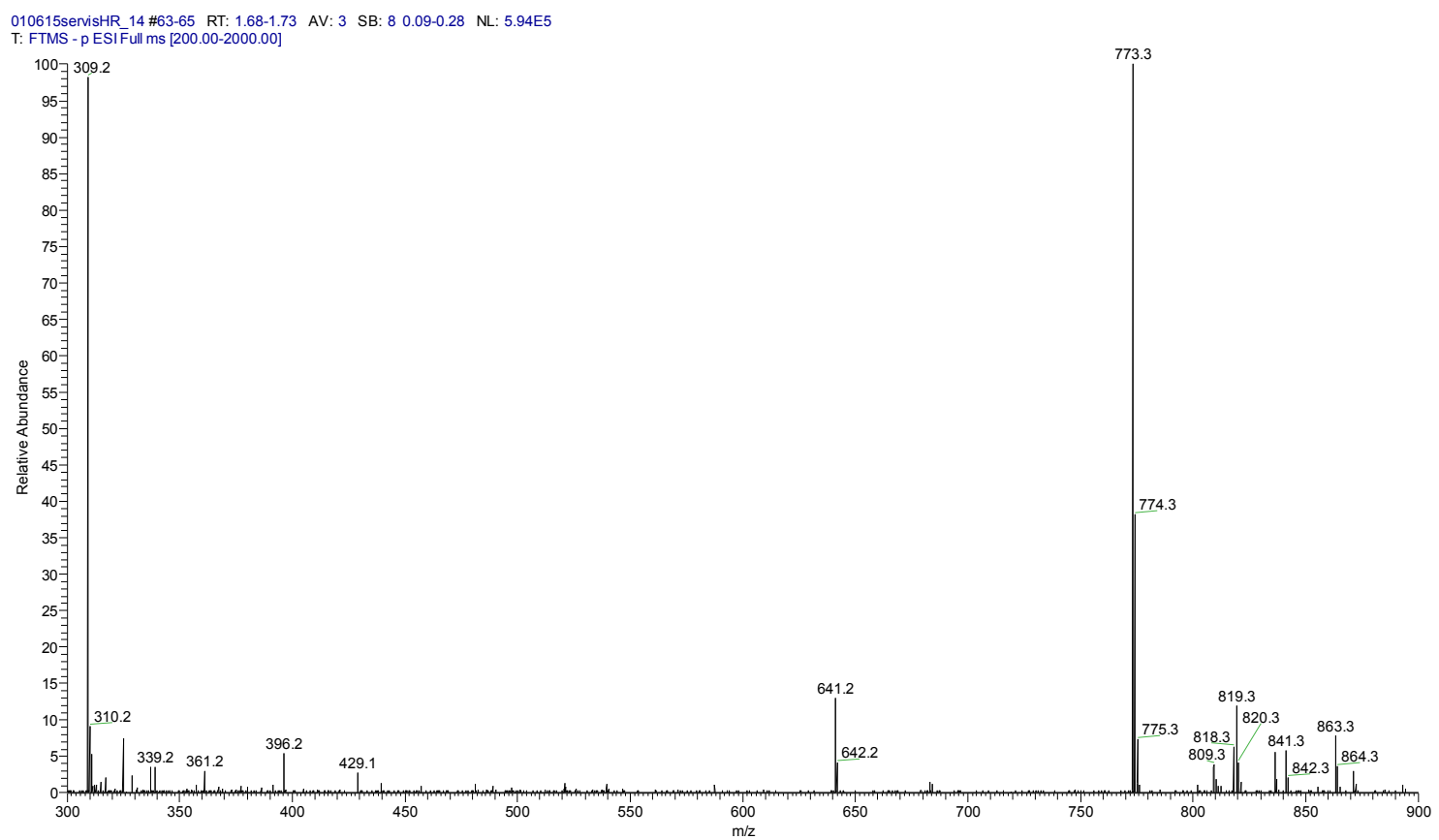

Figure S19. ESI-MS - spectrum of 3,7-di-O-benzyl-23-O-(tert-butyldimethylsilyl)-2,3-dehydrosilybin (15)<smiles>COCOc1cc(C(=O)Oc2ccc([C@H]3Oc4cc(-c5oc6cc(O)cc(O)c6c(=O)c5O)ccc4O[C@@H]3CCO[Si](C)(C)C(C)(C)C)cc2OC)cc(OCOC)c1OCOC</smiles>

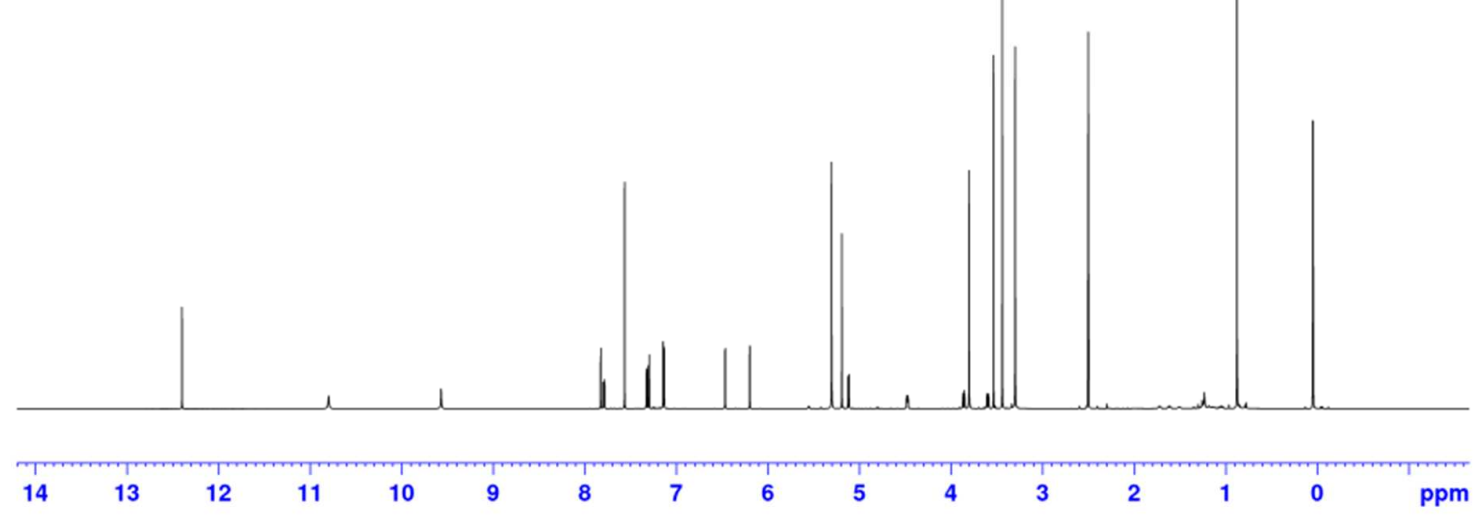

Figure S20. ${ }^{1} \mathrm{H}$ NMR spectrum $\left(700.13 \mathrm{MHz}, 303.2 \mathrm{~K}\right.$, DMSO- $\left.d_{6}\right)$ of 23-O-tert-butyldimethylsilyl-20-O$\left[\left(3^{\prime}, 4^{\prime}, 5^{\prime}\right.\right.$-tri-O-methoxymethyl)galloyl $]-2,3$-dehydrosilybin (18) 


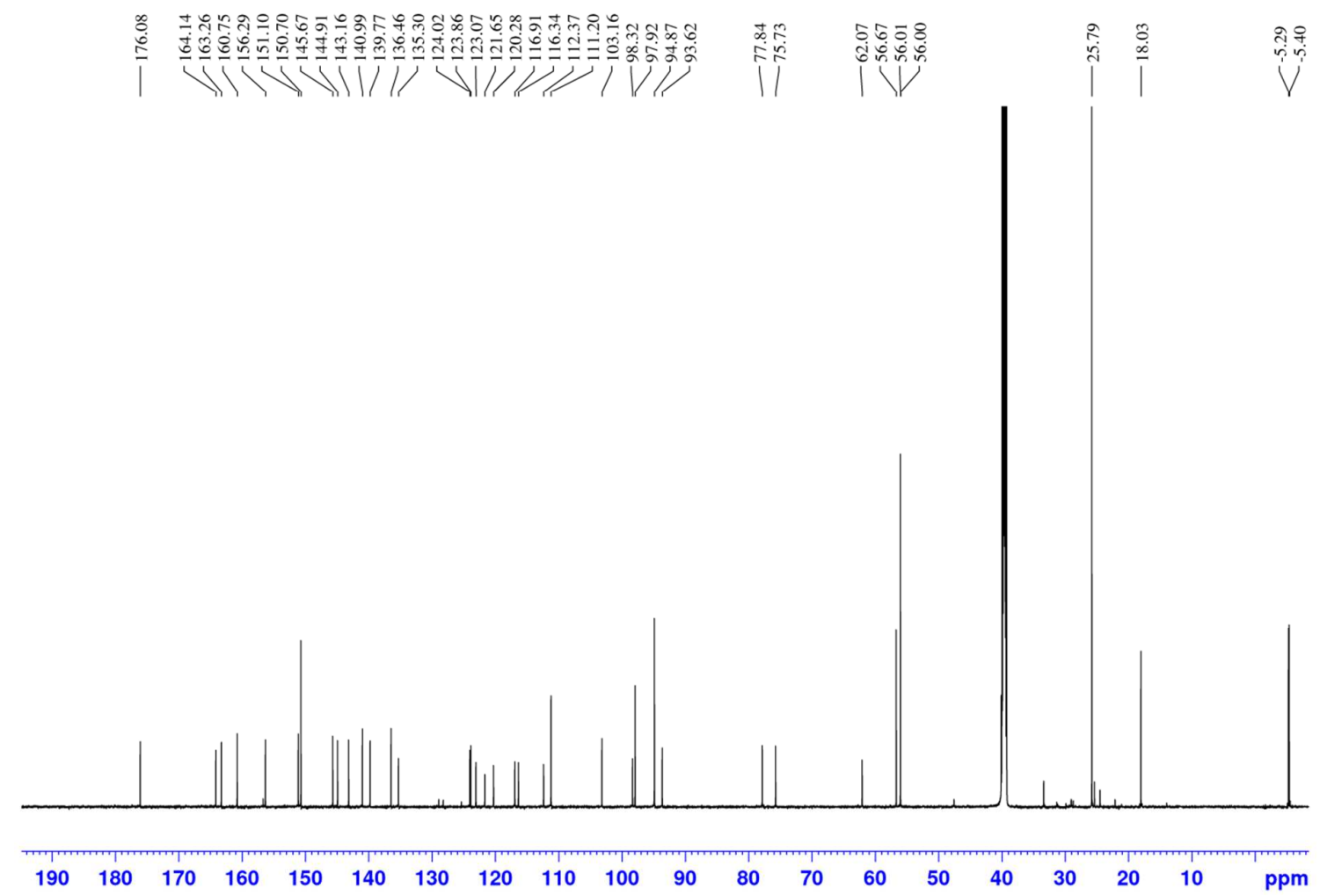

Figure S21. ${ }^{13} \mathrm{C}$ NMR spectrum $\left(176.05 \mathrm{MHz}, 303.2 \mathrm{~K}\right.$, DMSO- $\left.d_{6}\right)$ of 23-O-tert-butyldimethylsilyl-20-O$\left[\left(3^{\prime}, 4^{\prime}, 5^{\prime}\right.\right.$-tri-O-methoxymethyl)galloyl $]-2,3-$ dehydrosilybin (18)

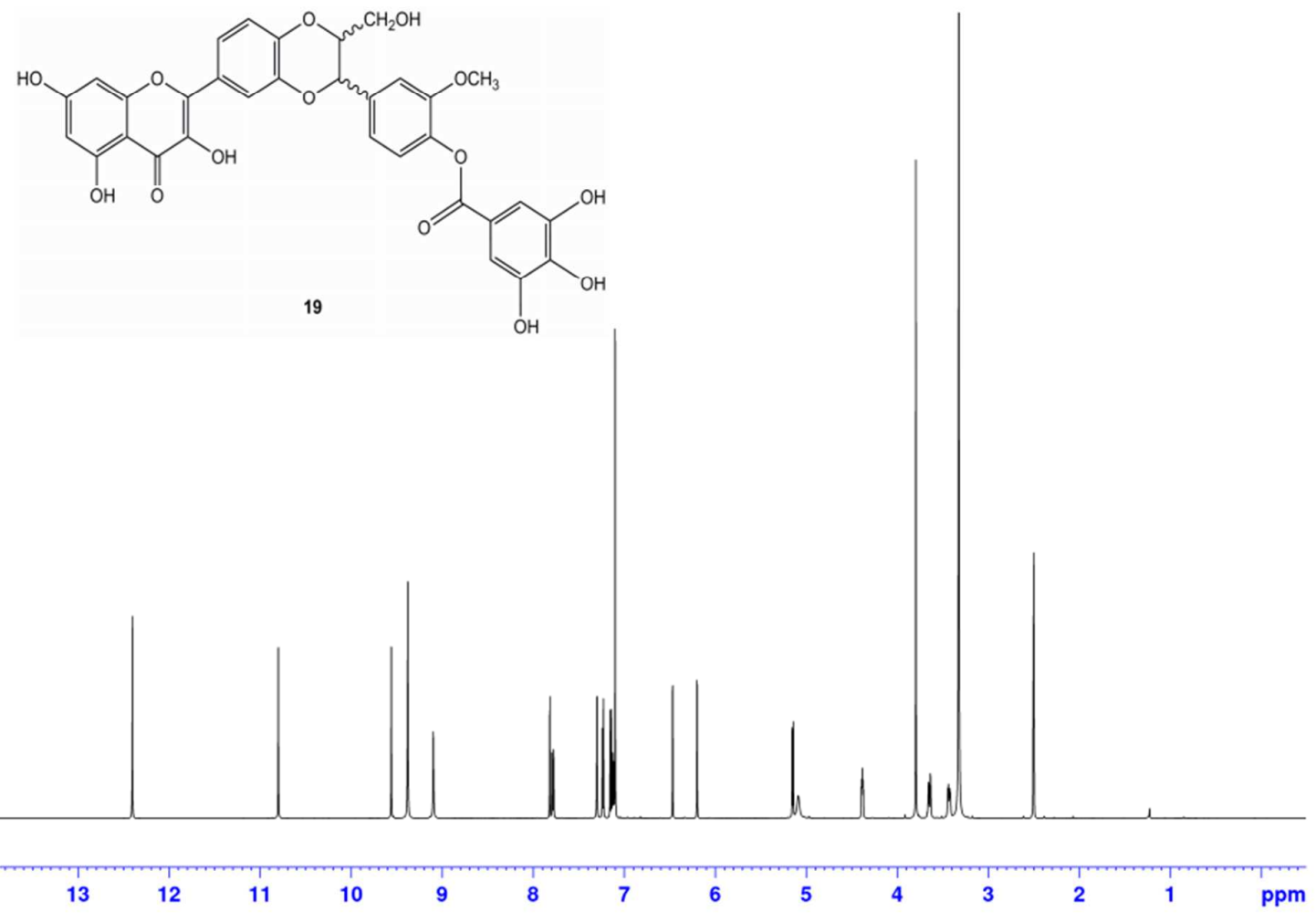

Figure S22. ${ }^{1} \mathrm{H}$ NMR spectrum (600.23 MHz, 303.2 K, DMSO- $d_{6}$ ) of 20-0-galloyl-2,3-dehydrosilybin (19) 


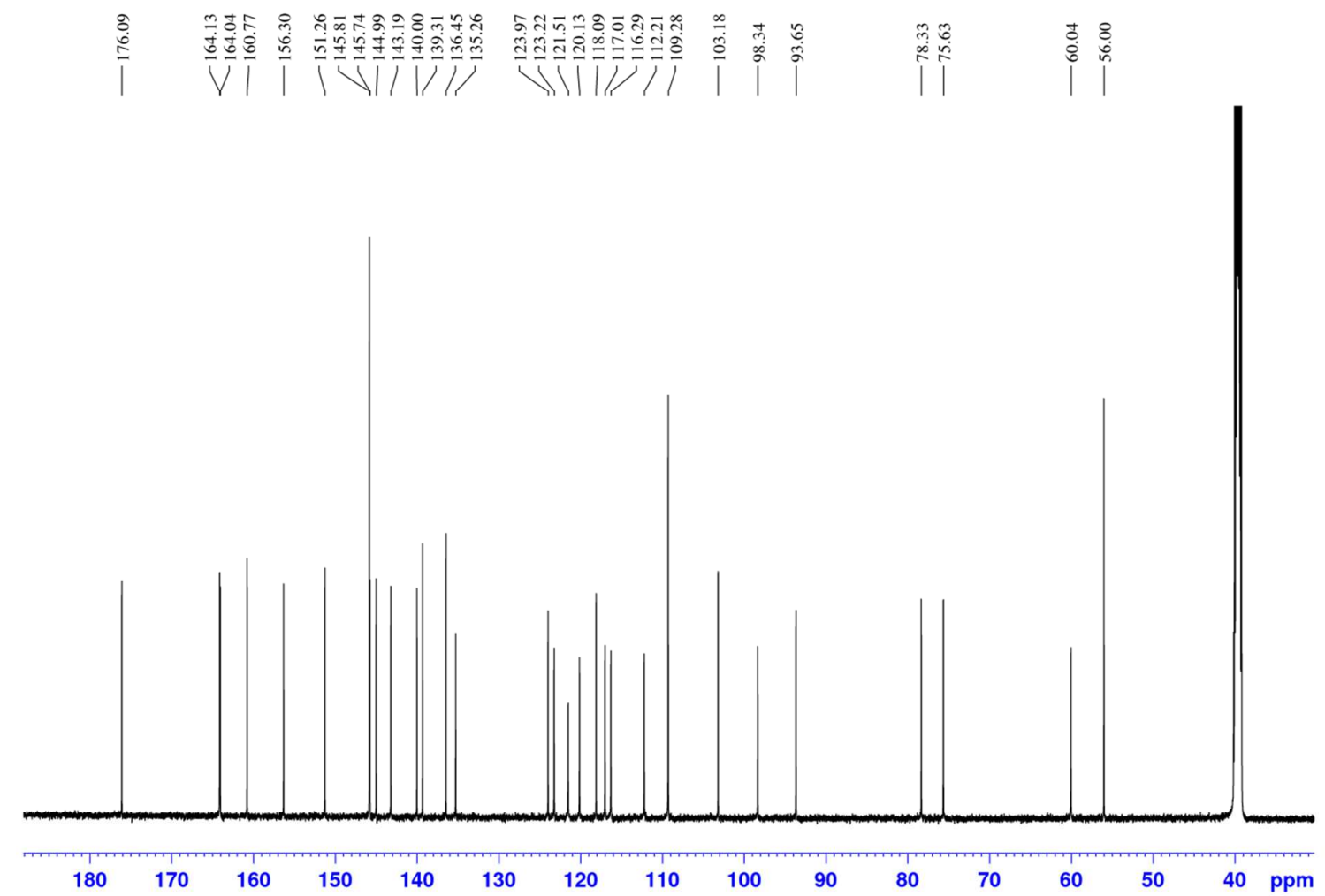

Figure S23. ${ }^{13} \mathrm{C}$ NMR spectrum (150.93 MHz, 303.2 K, DMSO- $d_{6}$ ) of 20-O-galloyl-2,3-dehydrosilybin (19)

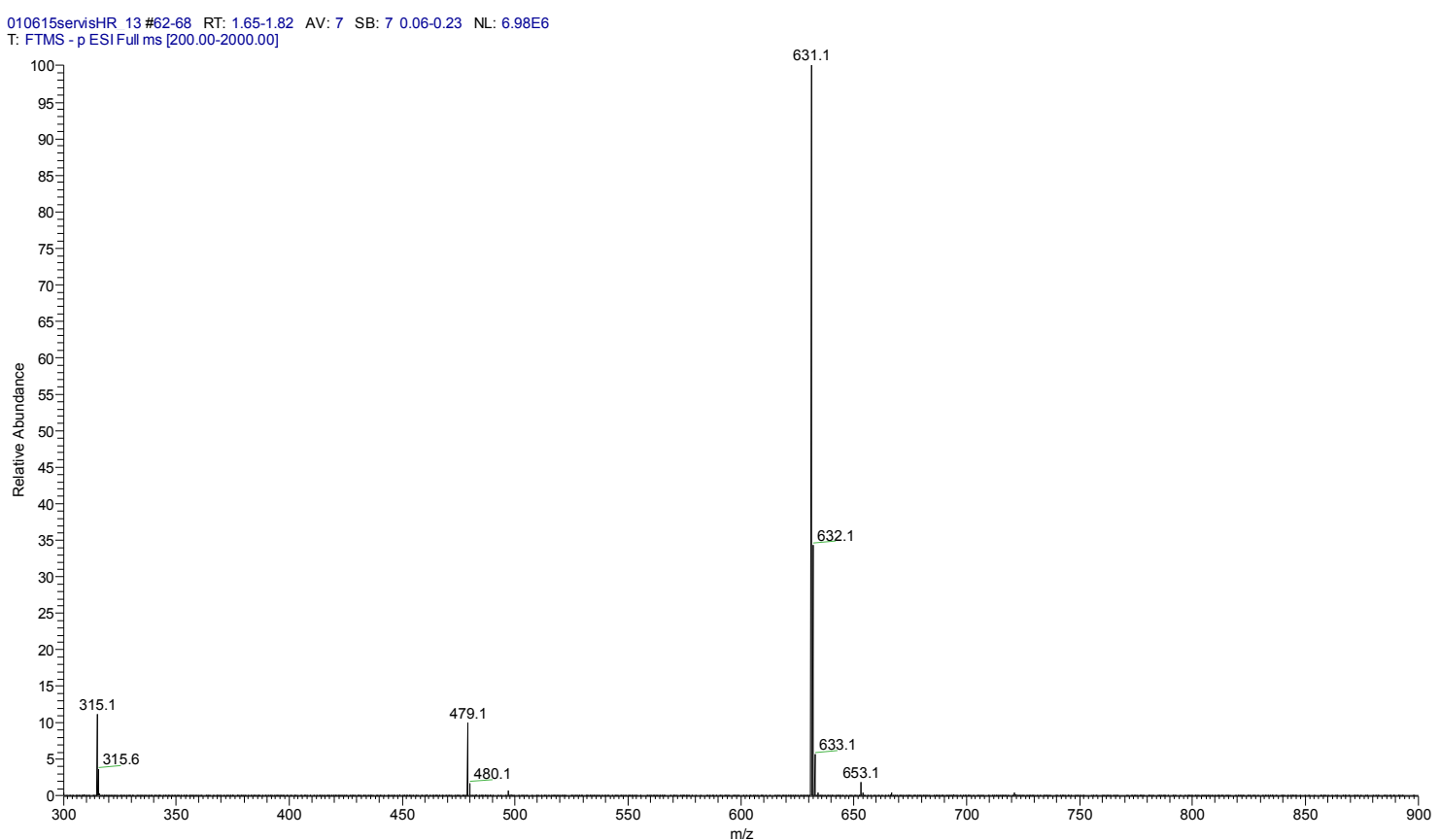

Figure S24. ESI-MS - spectrum of 20-O-galloyl-2,3-dehydrosilybin (19) 


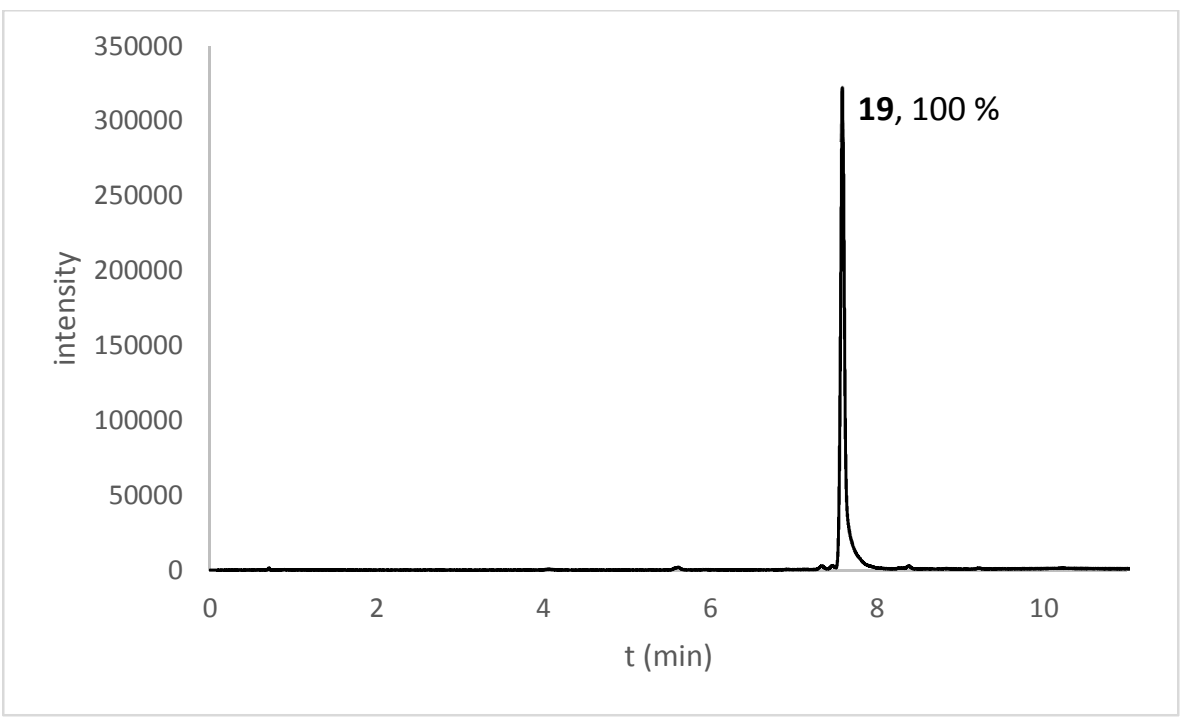

Figure S25. HPLC chromatogram of 20-O-galloyl-2,3-dehydrosilybin (19)<smiles>COCOc1cc(C(=O)O)cc(OCOC)c1OCOC</smiles>

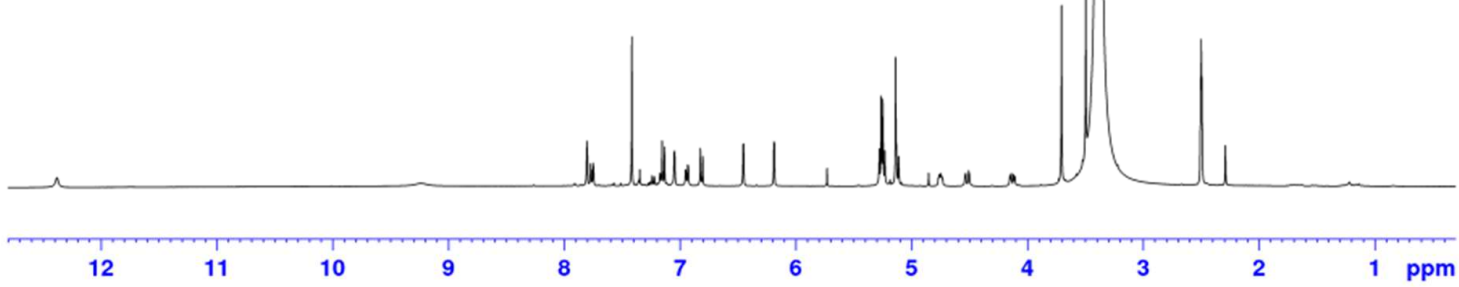

Figure S26. ${ }^{1} \mathrm{H}$ NMR spectrum $\left(399.87 \mathrm{MHz}, 303.2 \mathrm{~K}, \mathrm{DMSO}-\mathrm{d}_{6}\right)$ of $23-O-\left(3^{\prime}, 4^{\prime}, 5^{\prime}\right.$-tri- $O$ methoxymethylgalloyl)-2,3-dehydrosilybin (21) 


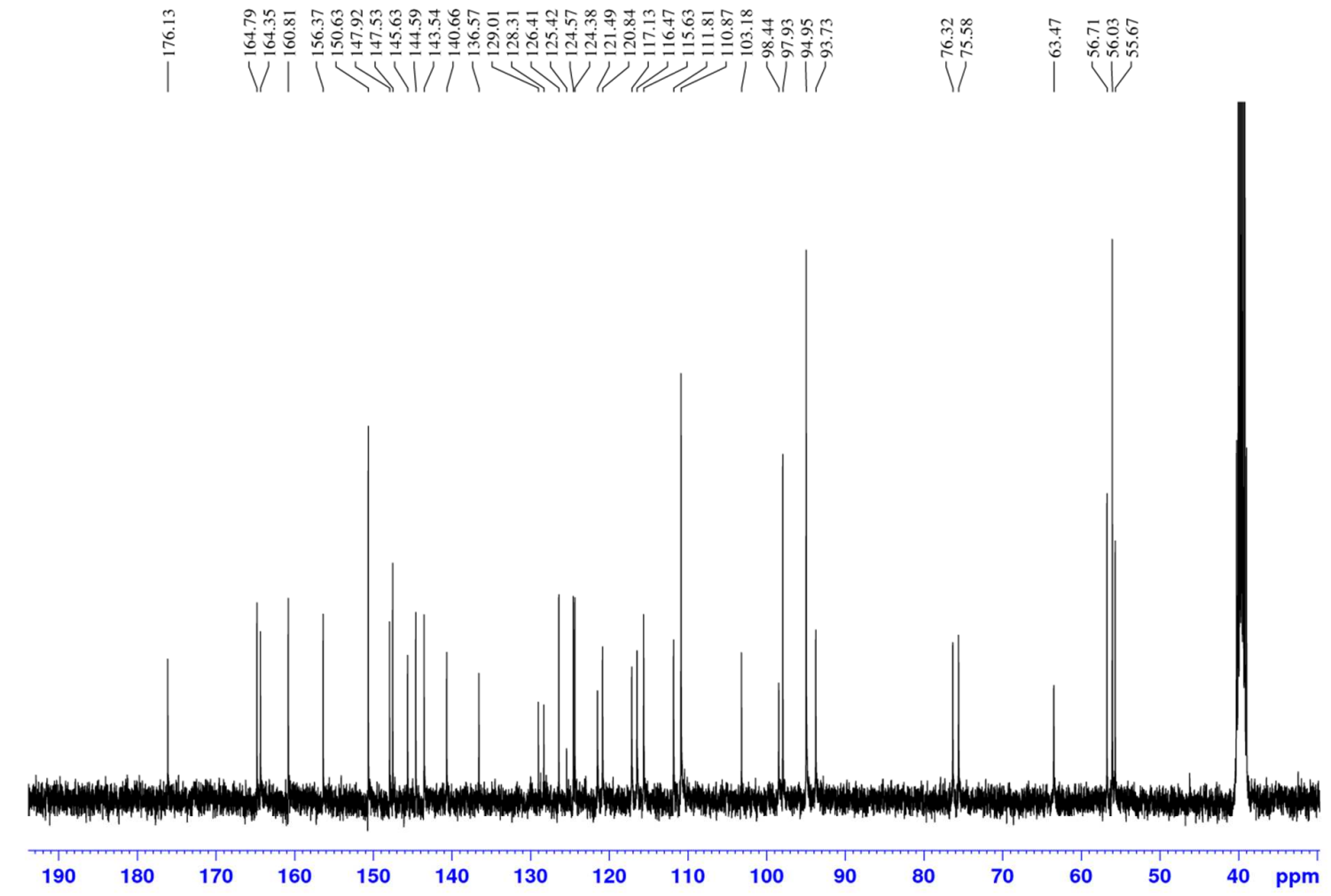

Figure S27. ${ }^{13} \mathrm{C}$ NMR spectrum $\left(100.56 \mathrm{MHz}, 303.2 \mathrm{~K}, \mathrm{DMSO}-\mathrm{d}_{6}\right)$ of 23-O-(3',4',5'-tri- $O$ methoxymethylgalloyl)-2,3-dehydrosilybin (21)

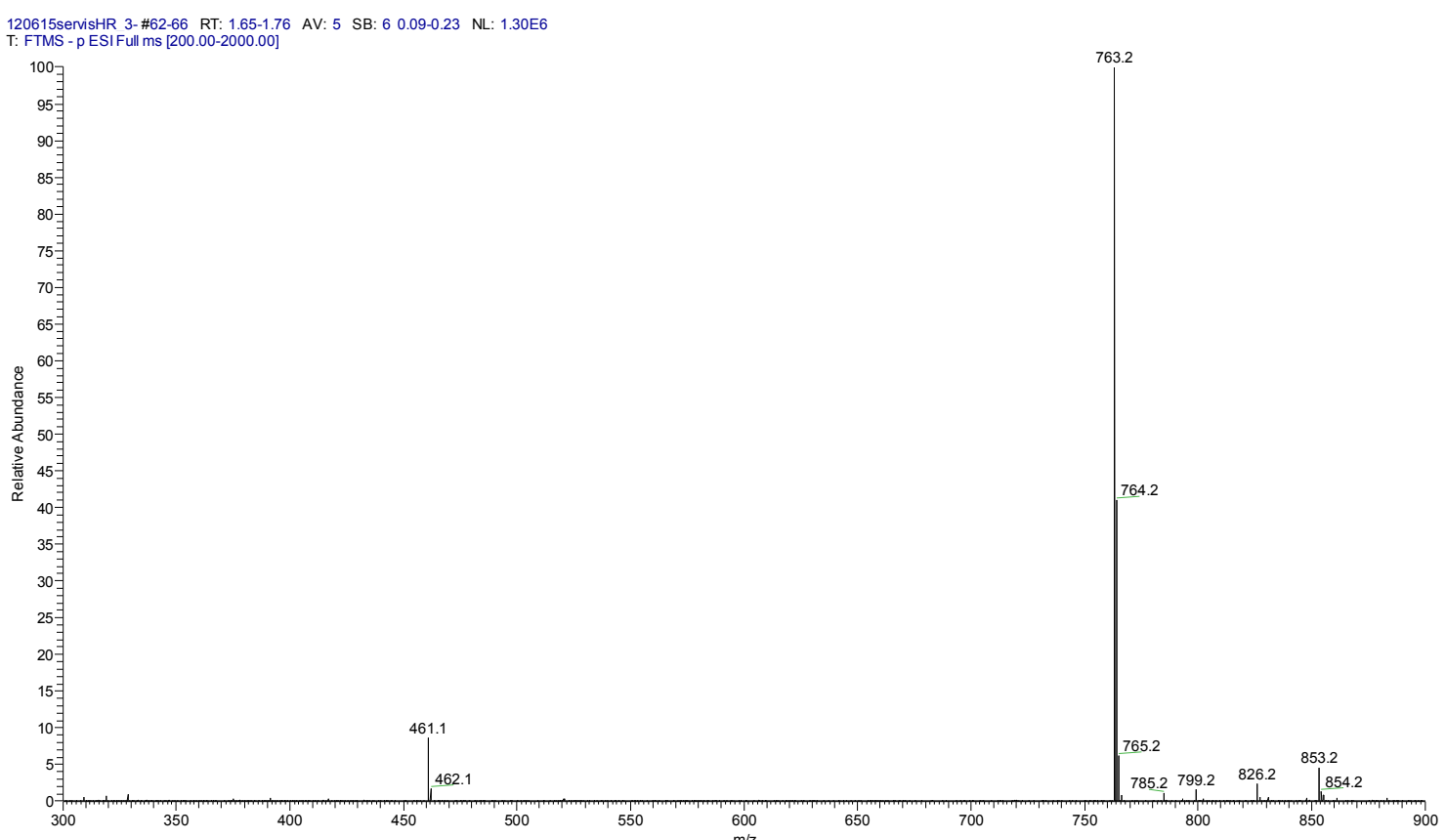

Figure S28. ESI-MS - spectrum of 23-O-(3',4',5'-tri-O-methoxymethylgalloyl)-2,3-dehydrosilybin (21) 


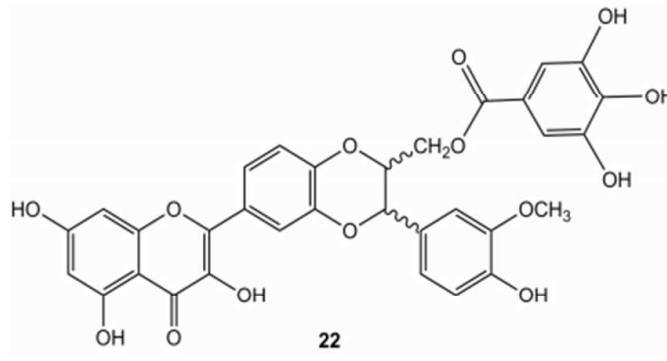

22

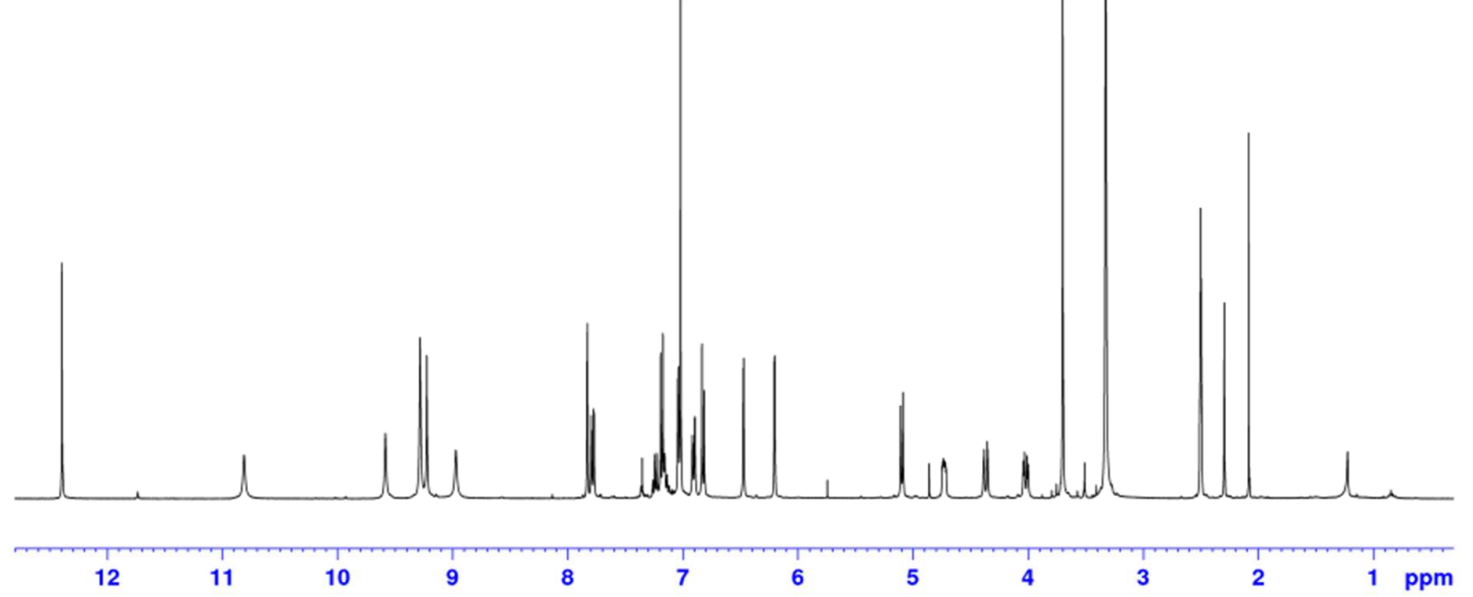

Figure S29. ${ }^{1} \mathrm{H}$ NMR spectrum $\left(399.87 \mathrm{MHz}, 303.2 \mathrm{~K}\right.$, DMSO- $\left.d_{6}\right)$ of 23-O-galloyl-2,3-dehydrosilybin (22)
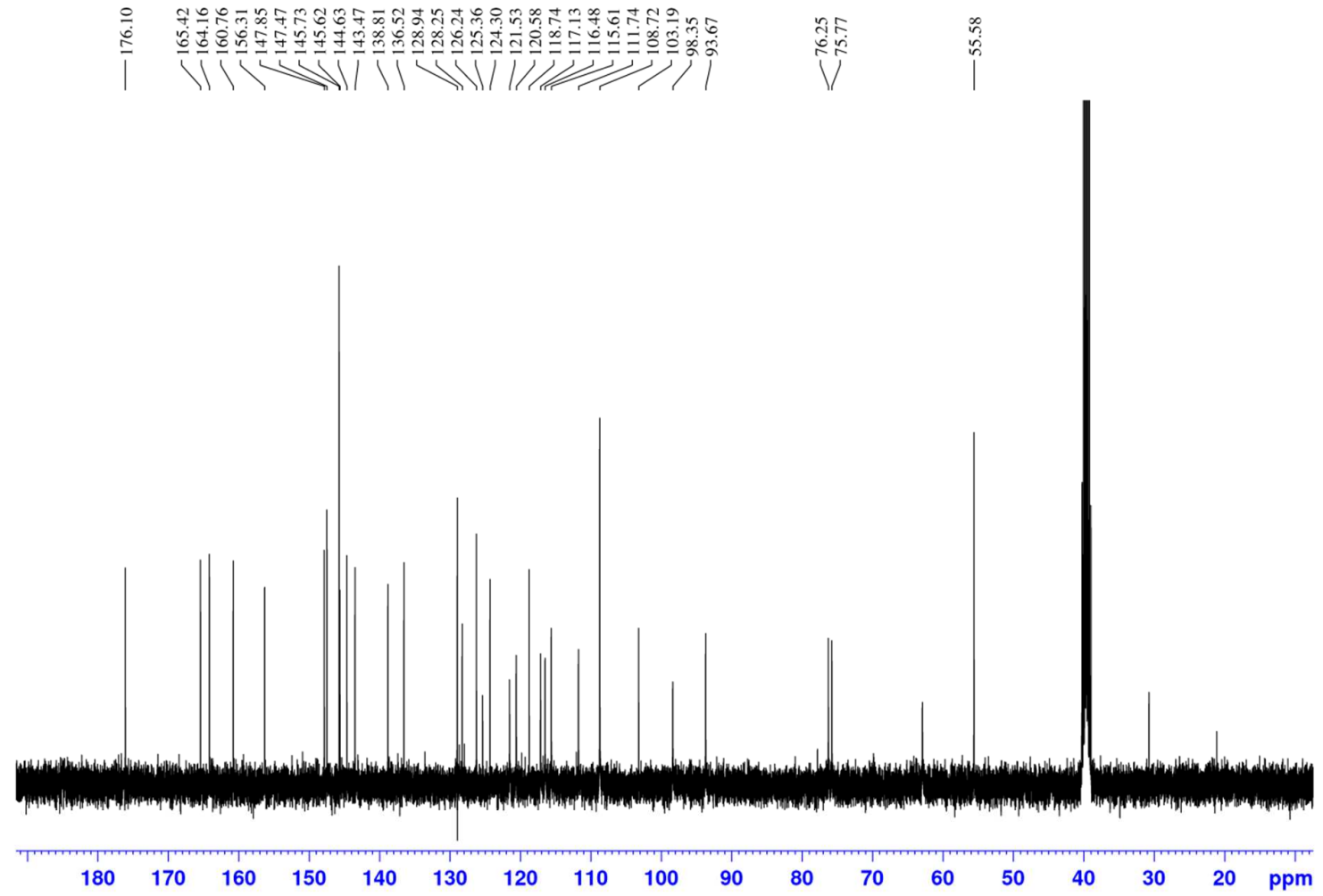

Figure S30. ${ }^{13} \mathrm{C}$ NMR spectrum (100.56 MHz, 303.2 K, DMSO- $d_{6}$ ) of 23-O-galloyl-2,3-dehydrosilybin (22) 


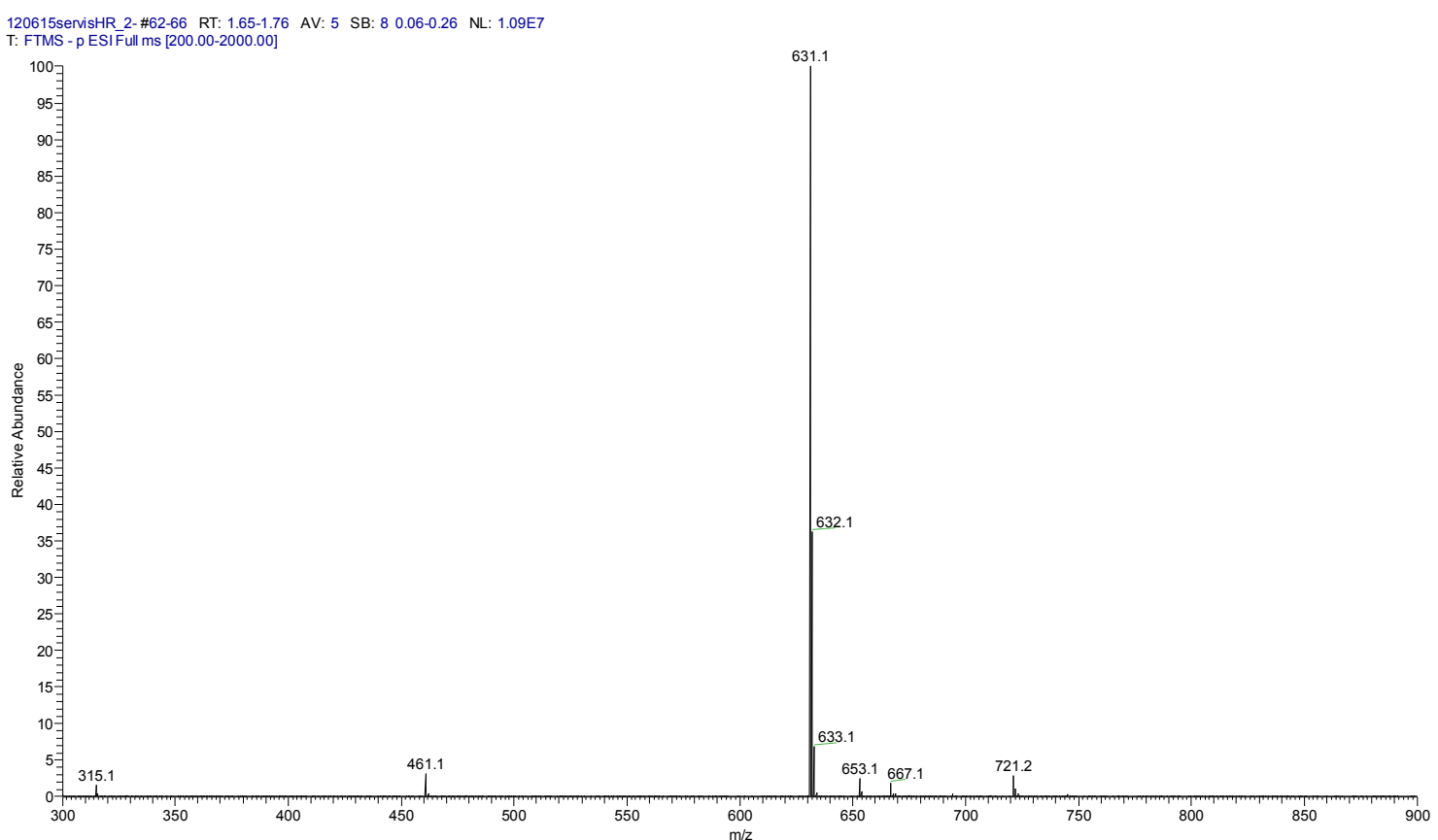

Figure S31. ESI-MS - spectrum of 23-O-galloyl-2,3-dehydrosilybin (22)

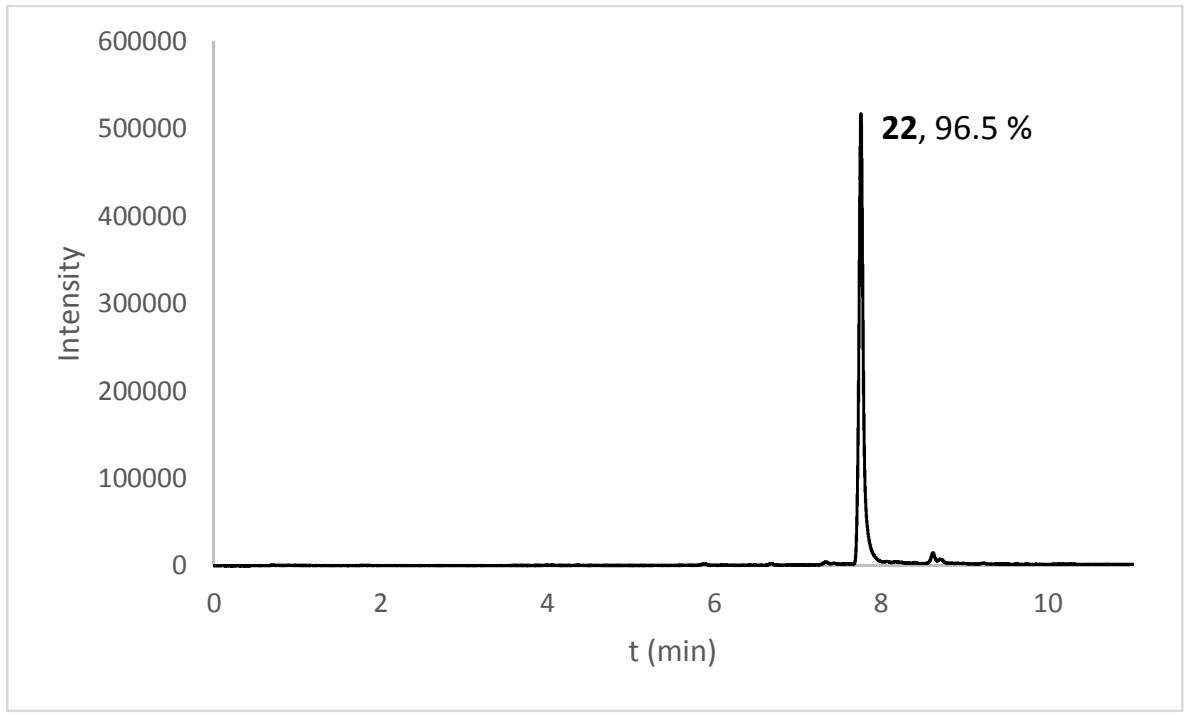

Figure S32. HPLC chromatogram of 23-O-galloyl-2,3-dehydrosilybin (22) 